Portland State University

PDXScholar

\title{
Adaptation of Classical Tidal Harmonic Analysis to Nonstationary Tides, with Application to River Tides
}

\author{
Pascal Matte \\ Institut National de la Recherche Scientifique \\ David A. Jay \\ Portland State University, djay@pdx.edu \\ Edward D. Zaron \\ Portland State University, ezaron@pdx.edu
}

Follow this and additional works at: https://pdxscholar.library.pdx.edu/cengin_fac

Part of the Civil Engineering Commons, and the Environmental Engineering Commons Let us know how access to this document benefits you.

\section{Citation Details}

Matte, P., Jay, D. A., \& Zaron, E. D. (2013). Adaptation of Classical Tidal Harmonic Analysis to Nonstationary Tides, with Application to River Tides. Journal Of Atmospheric \& Oceanic Technology, 30(3), 569-589.

This Article is brought to you for free and open access. It has been accepted for inclusion in Civil and Environmental Engineering Faculty Publications and Presentations by an authorized administrator of PDXScholar. Please contact us if we can make this document more accessible: pdxscholar@pdx.edu. 


\title{
Adaptation of Classical Tidal Harmonic Analysis to Nonstationary Tides, with Application to River Tides
}

\author{
Pascal MatTe,* David A. JAY, AND Edward D. Zaron \\ Department of Civil and Environmental Engineering, Portland State University, Portland, Oregon
}

(Manuscript received 21 December 2011, in final form 29 October 2012)

\begin{abstract}
One of the most challenging areas in tidal analysis is the study of nonstationary signals with a tidal component, as they confront both current analysis methods and dynamical understanding. A new analysis tool has been developed, NS_TIDE, adapted to the study of nonstationary signals, in this case, river tides. It builds the nonstationary forcing directly into the tidal basis functions. It is implemented by modification of T_TIDE; however, certain concepts, particularly the meaning of a constituent and the Rayleigh criterion, are redefined to account for the smearing effects on the tidal spectral lines by nontidal energy. An error estimation procedure is included that constructs a covariance matrix of the regression coefficients, based on either an uncorrelated or a correlated noise model. The output of NS_TIDE consists of time series of subtidal water levels [mean water level (MWL)] and tidal properties (amplitudes and phases), expressed in terms of external forcing functions. The method was tested using records from a station on the Columbia River, $172 \mathrm{~km}$ from the ocean entrance, where the tides are strongly altered by river flow. NS_TIDE hindcast explains $96.4 \%$ of the signal variance with a root-mean-square error of $0.165 \mathrm{~m}$ obtained from 288 parameters, far better than traditional harmonic analysis $(38.5 \%, 0.604 \mathrm{~m}$, and 127 parameters). While keeping the benefits of harmonic analysis, its advantages compared to existing tidal analysis methods include its capacity to distinguish frequencies within tidal bands without losing resolution in the time domain or data at the endpoints of the time series.
\end{abstract}

\section{Introduction}

Surface tides in the deep ocean are predictable at most locations because they are nearly periodic. There are many situations, however, in which the observed tides (a combination of barotropic and baroclinic waves) are modulated by nontidal processes, some of which occur in the tidal frequency band. Perturbations of the tidal signal may arise from variations in external forcingeither oceanic, meteorological, hydrologic, or climatic. They may be abrupt and aperiodic (with energy at periods of a few hours to a few days), quasi periodic (e.g., a diurnal sea breeze or annual river flow cycle), long term (periods of years to decades), or secular (without

\footnotetext{
* Current affiliation: Centre Eau Terre Environnement, Institut National de la Recherche Scientifique, Quebec, Quebec, Canada.

Corresponding author address: Pascal Matte, Centre Eau Terre Environnement, Institut National de la Recherche Scientifique, 490 de la Couronne Street, Quebec QC G1K 9A9, Canada. E-mail: pascal.matte@ete.inrs.ca
}

an apparent period within the length of the available tidal record). Also, morphological modifications leading to changes in bed friction, surface slope, and/or vegetation may all alter tidal properties (Amin 1983, 1985; Godin 1985; DiLorenzo et al. 1993; Horsburgh and Wilson 2007; Jay 2009; Jay et al. 2011). Such circumstances present a severe challenge for conventional tidal prediction methods, but also an opportunity for dynamical inquiry, provided that adequate analytical tools are available. Our purpose here is to generalize traditional harmonic analysis (HA) to allow for the study of nonstationary tides. We accomplish this by building the forcing that causes the nonstationary tidal response into the basis functions employed in the analysis. To demonstrate the utility of the method, we apply it to the problem of river tides, because they are "conceptually the simplest non-stationary tidal process, and the only one for which both ample data and a detailed theoretical analysis are available" (Jay and Flinchem 1997, p. 5705).

Tides in rivers do not respond simply to astronomical forcing, but are the result of nonlinear interactions of the 
oceanic tide with channel geometry, bottom friction, and river flow. As a consequence, the tide is increasingly distorted and damped as it propagates upriver (Godin 1985; Aubrey and Speer 1985; Speer and Aubrey 1985; Parker 1991; Godin 1999). This leads to asymmetries in the river tide that are manifested by unequal duration of ebb and flood, resulting in irregularities in the timing and height of high and low water (Godin 1984, 1999; Nidzieko 2010). Tidal monthly oscillations of mean water level (MWL) induced by the neap-spring cycle also increase in strength upstream. The amplitude of this fortnightly tide eventually surpasses that of the dominant diurnal and semidiurnal tides, leading to the reversal of the relative levels of mean low water at spring and neap tides (LeBlond 1979, 1991; Gallo and Vinzon 2005.

Tidal motion in shallow rivers is best represented by a first-order differential equation with only one (incident) wave, which diffuses in from time-varying forcing conditions at the river mouth (LeBlond 1978). The dynamic balance is between surface slope and friction, whose effects on subtidal and tidal water level variations can be captured in analytical solutions of the one-dimensional St. Venant equations. The solutions are based on a decomposition of the nonlinear friction term (e.g., Dronkers 1964; Godin 1999) into contributions caused by external parameters and nonlinear interactions. Simple regression models, exploiting the results of tidal analysis [whether HA, continuous wavelet transform (CWT), or some other form], can be used to identify and predict the relative importance of these contributions to local tidal dynamics.

Jay and Flinchem (1997) and Godin (1999) were among the first ones to show the predicting potential of such models. Jay and Flinchem (1997) obtained analytical predictions of the response of the low-frequency, diurnal, semidiurnal, and quarterdiurnal tidal elevations to variations in river flow and successfully compared them to CWT analyses of tidal height records in the Columbia River. Godin (1999) demonstrated that variations in MWL and tidal properties at upriver stations in the St. Lawrence River can be predicted from forcing conditions (i.e., tidal constituents amplitudes, tidal range, and/or river discharge). He further showed the correlation between river discharge and the amplitude and phase variations of the semidiurnal tide, the local tidal range, and the times of arrival of high and low water.

Kukulka and Jay $(2003 a, b)$ derived from a theory of river-tide propagation in convergent channels with strong friction (Jay 1991) a functional representation that uncouples the nonlinear interactions of fluvial tides and river stage (i.e., MWL). They used a CWT analysis of Columbia River tides to provide time series of stage, tidal amplitudes and phases, and tidal range, which they regressed against coastal tidal range and river flow. Their models agreed remarkably well with observations even at seaward stations, achieving better agreement with observation than hindcasts available from conventional HA. Elaborating on this work, Jay et al. (2011) adapted the method to model tidal extremathat is, lower low water (LLW) and higher high water (HHW) - as a function of river flow and external tidal forcing.

Buschman et al. (2009) presented a method to analyze subtidal water levels in tidal rivers. Unlike Kukulka and Jay's (2003a,b) models, they used Godin's approximation of the friction term (Godin 1999), rather than the Tschebyschev polynomial approach (Dronkers 1964), to derive a new expression for subtidal friction, and successfully applied their model on the Berau River (Indonesia). Together, these studies have shown the potential of including the contributions of external tidal and fluvial forcing to improve tide predictions in the upper regions of rivers.

We introduce here a generalization of classical tidal HA to nonstationary tidal records. An analytical model, adapted from Kukulka and Jay (2003a,b) and Jay et al. (2011), is directly embedded into the HA basis functions to allow the treatment of tidal-fluvial interactions. Although we focus on the problem of river tides, the method is applicable in any situation in which tides are modulated by a quantifiable nontidal process. We seek an analysis tool that is able to

- embed an analytical model of nontidal perturbations into the HA basis functions;

- provide time series of subtidal and tidal properties (MWL, amplitudes, and phases), expressed in terms of external forcing;

- distinguish frequencies within tidal species;

- improve the predictive power of HA in highly nonlinear environment (e.g., upstream reaches of tidal rivers);

- evaluate errors in constituent properties.

HA is possibly the most widely used approach in tidal analysis. It determines the phase and amplitude coefficients of a priori known frequencies via a least squares fitting procedure. Each tidal constituent is represented by a sine wave whose frequency is derived from the tidal potential and nonlinear interactions. HA relies, however, on the assumptions that the analyzed signal is stationary and that constituents are independent. For a nonstationary signal, it provides approximate values of tidal constituent properties, but with no information regarding their evolution in time and the underlying dynamics involved (Jay and Flinchem 1997, 1999). 
Several methods have been applied to time series to extract information on transient tidal processes. They are generally devised to transform the content of a signal in the time domain into frequency information that expresses the amplitude and phase behavior of the input as a function of both frequency and time [for an overview of existing methods, see, e.g., Jay and Kukulka (2003) and Parker (2007)]. While these methods allow a representation of the modulations induced by nonstationary processes, their success has been limited either by a loss of resolution of constituents within a tidal band [e.g., short-term HA (STHA), complex demodulation, CWT, empirical mode decomposition] or by their inability to treat signals with strong nonlinearities or sharp variations in nontidal forcing (e.g., STHA, response method, species concordance method).

Our implementation of HA for nonstationary records, NS_TIDE, is carried out through modifications of the T_TIDE package in MATLAB (Pawlowicz et al. 2002; Leffler and Jay 2009). In common with traditional HA, our method assumes that the analysis frequencies are a priori known, even if some of them are nontidal. However, changes are made to make the method suitable for the analysis of nonstationary signals. We first rethink the concept of harmonic constants because constituent amplitudes and phases for any station are modulated by time-dependent nontidal forcing. Second, for the assumption of prior knowledge of tidal frequencies to be maintained, new criteria are defined to select and determine the significance of selected constituents.

This paper is structured as follows. The models implemented in NS_TIDE are presented in section 2, together with a redefinition of the Rayleigh criterion and of the error estimation. In section 3 , the method is applied to tidal records for the lower Columbia River and compared to results from traditional HA. Section 4 discusses and summarizes the results. The appendix explains details regarding the analysis method.

\section{Model description}

\section{a. Harmonic models}

HA methods originated in the late nineteenth century (e.g., Darwin 1891) and were given a structure based on a modern understanding of the tidal potential by Doodson (1921). Several contemporary tidal analysis codes stem from Godin's (1972) reformulation of Doodson's work, in particular the FORTRAN code of Foreman (1977) and the MATLAB code of T_TIDE (Pawlowicz et al. 2002). HA codes model tidal heights $h\left(t_{j}\right)$ as a function of time $t_{j}$, with known tidal constituent frequencies $\sigma_{k}$ and unknown amplitudes $b_{0}, b_{1, k}$, and $b_{2, k}$; thus,

$$
h\left(t_{j}\right)=b_{0}+\sum_{k=1}^{n}\left[b_{1, k} \cos \left(\sigma_{k} t_{j}\right)+b_{2, k} \sin \left(\sigma_{k} t_{j}\right)\right] .
$$

Improvements to traditional harmonic methods have been made in the recent years in the inverse technique used and the estimation of confidence limits. Leffler and Jay (2009) incorporated robust estimators (Huber 1996) via iteratively reweighted least squares (IRLS) into T_TIDE to reduce the influence of nontidal variation on the overall fit by downweighting outliers. Foreman et al. (2009) enhanced HA by embedding nodal and astronomical argument corrections and multiple inference calculations directly in the least squares matrix, thereby removing the need for postfit adjustments. The most direct consequence of this is that corrections and inferences influence all constituents included in the analysis rather than the specific constituents actually corrected. These improvements are included in Foreman's FORTRAN code, as well as in the UTide MATLAB functions (Codiga 2011). NS_TIDE is implemented as a modification to T_TIDE. It is similar to Foreman et al. (2009), in that the models describing stage and tidal-fluvial variations are directly embedded into the basis functions of the matrix system to be solved. It differs from previous software in its conception of tides as fundamentally nonstationary, in its implementation of the Rayleigh criterion, and in its error analysis.

\section{b. Stage and tidal-fluvial models}

The conceptual approach we advance here is quite general with respect to the physical processes that perturb the tidal "constants." To provide a concrete example, we apply the method to river tides, using the framework provided by Kukulka and Jay (2003a,b) and Jay et al. (2011), summarized below. Kukulka and Jay (2003a,b) expressed the bed stress $\tau_{B}=\rho C_{D}|U| U$ using the Tschebyschev polynomial representation of $|U| U$ provided by Dronkers (1964), where $U$ is velocity, $\rho$ is water density, and $C_{D}$ is the drag coefficient. The bed stress $\tau_{B}$ controls the complex wavenumber $q=k+\mathrm{i} r$ and, therefore, governs tidal propagation; here, $k$ is the wavenumber and $r$ is the damping modulus. For the critical convergence regime (Jay 1991) where tidal and fluvial flows are of similar magnitude and convergence is moderate, $k=-r$ and $q=(\mathrm{i}-1) r$. Under these conditions, Kukulka and Jay (2003a) derived models for constituent amplitude (or tidal range) ratios between a station of interest and a reference station in terms of the damping modulus $r$ : 


$$
\begin{aligned}
\frac{\zeta(x)}{\zeta_{0}} & =e^{-i q\left(x-x_{0}\right)}, \\
\ln \left[\frac{|\zeta(x)|}{\left|\zeta_{0}\right|}\right] & =+r\left(x-x_{0}\right),
\end{aligned}
$$

where $\zeta(x)$ is a tidal property (amplitude or range) at position $x$ and $\zeta_{0}$ is the same property at the reference station at position $x_{0}$. The phase difference $\Delta \phi$ is modeled by analogy to $\zeta: \Delta \phi=-k\left(x-x_{0}\right)+a_{0}^{\prime}$, where $a_{0}^{\prime}$ is an offset coefficient. Kukulka and Jay (2003a) used the Dronkers (1964) representation of $|U| U$ to parameterize Eq. (2b) in terms of external variables $Q$ and $R$ representing river flow and greater diurnal tidal range at the reference station, respectively, yielding

$$
\ln \left[\frac{|\zeta(x)|}{\left|\zeta_{0}\right|}\right]=a_{0}+a_{1} Q^{p}+a_{2} \frac{R^{2}}{Q^{0.5}}
$$

where $\left(x-x_{0}\right)$ is assumed fixed and absorbed into the parameters, and $p=1$.

The coefficient $a_{0}$ in Eq. (3) is primarily determined by the convergence or divergence of the channel cross section, while $a_{1}$ represents nonlinear interactions with river flow. Kukulka and Jay (2003a) assumed that river flow $Q$ should appear linearly in Eq. (3). This simplifies the actual nonlinear response of tidal parameters to river flow in Jay (1991) without loss of accuracy (Kukulka and Jay 2003a). Moreover, the appearance of $Q$ in Eq. (3) is itself a simplification-in theory, $U=Q / A(Q)$ should be used, where $A(Q)$ is the crosssectional area. The variation of $A$ with $Q$ can cause deviations of the exponent $p$ from unity depending on channel geometry at the site and seaward of the tide gauge, through which the tidal wave propagates. While $p$ may be kept constant by adding additional regression terms that represent the variation in $A$ with $Q$, this has an unfavorable effect on model confidence limits. A pragmatic solution is to optimize the exponents by station, as in Jay et al. (2011).

The last term in Eq. (3) represents the effects of nonlinear tidal-fluvial interactions due to neap-spring variability, whose importance decreases as river flow increases. It is responsible for the tidal monthly changes in MWL and tidal properties, attributable to frictional interactions. In theory, this interaction is quadratic in $R$, but only for stations where river flow and tidal currents are comparable in strength. Again, optimal exponents can be obtained to account for deviations from theory (e.g., Jay et al. 2011).

Equation (3) is dimensionally inhomogeneous, in that the values of $a_{1}$ and $a_{2}$ depend on the units of measurement, and their units depend on the exponents of $Q$ and $R$. These constants ought to involve the geometry of the river, including, for example, the crosssectional area, the convergence rate, the wetted perimeter, and possibly other factors. In other words, placing this formula into dimensionally homogenous form would involve explicitly writing the dependence of $a_{i}(i=1,2)$ and the exponents on the appropriate nondimensional parameters. These parameters will vary from place to place, and their values will often be unknown or uncertain. Rather than explicitly modeling these relationships, we have taken the pragmatic approach of adjusting the exponents and the coefficients to fit the data. And, interestingly enough, the sensitivity of the results to the exponents is little (see section 3 ).

Expressions such as Eq. (3) can be used to describe tidal amplitude, phase, or range variations in time. Kukulka and Jay (2003b) derived a similar expression to model mean river stage (MWL), with an additional term to account for variations in atmospheric pressure. Jay et al. (2011) further extended these functional representations to model LLW and HHW, which are quantities that combine river stage and tidal properties.

\section{c. Practical regression models}

The present analysis uses a functional representation derived from the latest version of the model by Jay et al. (2011), in which the exponents associated with the various terms in Eq. (3) are iteratively optimized to account for deviations from theory, due to the effects of timevarying channel geometries and variations in the ratio of river flow to tidal currents as a function of upriver distance. In practice, the variations in tidal parameters are small enough that the logarithm of the left-hand side of Eq. (3) can be replaced by a linear term, using the first term in the Taylor series expansion of the natural $\operatorname{logarithm}, \ln (1+\delta) \sim \delta$, where $\delta$ is the departure of the ratio in Eq. (3) from unity (cf. Jay et al. 2011).

Conceptually, we replace the constants $b_{0}, b_{1, k}$, and $b_{2, k}$ in Eq. (1) with functions of river flow $Q$ and greater diurnal tidal range $R$ at a convenient station removed from fluvial influence:

$$
\begin{aligned}
h\left(t_{j}\right)= & b_{0,0}\left[Q\left(t_{j}\right), R\left(t_{j}\right)\right] \\
& +\sum_{k=1}^{n}\left\{b_{1, k}\left[Q\left(t_{j}\right), R\left(t_{j}\right)\right] \cos \left(\sigma_{k} t_{j}\right)\right. \\
& \left.+b_{2, k}\left[Q\left(t_{j}\right), R\left(t_{j}\right)\right] \sin \left(\sigma_{k} t_{j}\right)\right\}, \\
b_{l, k}\left(t_{j}\right)= & a_{0, l, k}+a_{1, l, k} Q^{p}\left(t_{j}\right)+a_{2, l, k} \frac{R^{q}\left(t_{j}\right)}{Q^{r}\left(t_{j}\right)},
\end{aligned}
$$


where $h$ is tidal heights $(\mathrm{m}) ; t$ is time (s); $Q$ is river flow (thousands of $\mathrm{m}^{3} \mathrm{~s}^{-1}$ ); $R$ is the greater diurnal tidal range $(\mathrm{m}) ; p, q, r$ are the exponents for each station and frequency band; $a_{0}, a_{1}, a_{2}$ are the model parameters for each station and frequency; $b_{0}, b_{1, k}, b_{2, k}$ are the harmonic model amplitudes for each station and frequency; $j$ is the index for time $(j=1, m) ; k$ is the index for tidal constituents $(k=1, n) ; l$ is the index for coefficients $(l=$ $0,2) ; m$ is the number of observations in the time series; $n$ is the number of tidal constituents.

The $Q$ and $R$ time series are lagged to account for the distance separating the stations where they were measured and the station at which the analysis is performed. Time lags are determined by calculating the maximum correlation between $Q$ or $R$ and the filtered (i.e., low passed or range filtered) time series of measured tidal heights. A constant time lag representing the average time of propagation of the waves is applied to each forcing variable $Q$ and $R$. More complex lag functions could be used to better capture the varying propagation times as a function of river stage, but they are not currently implemented in NS_TIDE.

$$
\begin{aligned}
h\left(t_{j}\right)= & \underbrace{c_{0}+c_{1} Q^{p_{s}}\left(t_{j}\right)+c_{2} \frac{R^{q_{s}\left(t_{j}\right)}}{Q^{r_{s}\left(t_{j}\right)}}}_{\text {stage model or } s\left(t_{j}\right)} \\
& +\cdots \underbrace{\sum_{k=1}^{n}\left\{\left[d_{0, k}^{(c)}+d_{1, k}^{(c)} Q^{p_{f}}\left(t_{j}\right)+d_{2, k}^{(c)} \frac{R^{q_{f}\left(t_{j}\right)}}{Q^{r_{f}\left(t_{j}\right)}}\right] \cos \left(\sigma_{k} t_{j}\right)+\left[d_{0, k}^{(s)}+d_{1, k}^{(s)} Q^{p_{f}}\left(t_{j}\right)+d_{2, k}^{(s)} \frac{R^{q_{f}}\left(t_{j}\right)}{Q^{r_{f}}\left(t_{j}\right)}\right] \sin \left(\sigma_{k} t_{j}\right)\right\}}_{\text {tidal-fluvial model or } f\left(t_{j}\right)},
\end{aligned}
$$

To separately include the influence of multiple rivers and tidal inlets, the second and third terms of Eq. (4b) are generalized as follows (indices $l$ and $k$ are dropped for clarity):

$$
\begin{gathered}
a_{1} Q^{p}\left(t_{j}\right) \Rightarrow \sum_{u=1}^{n_{Q}} a_{1 u} Q_{u}^{p_{u}}\left(t_{j}\right), \\
a_{2} \frac{R^{q}\left(t_{j}\right)}{Q^{r}\left(t_{j}\right)} \Rightarrow \sum_{v=1}^{n_{R}} a_{2 v} \frac{R_{v}^{q_{v}}\left(t_{j}\right)}{\left[\sum_{u=1}^{n_{Q}} Q_{u}\left(t_{j}\right)\right]^{r_{v}}},
\end{gathered}
$$

where $u$ and $v$ are indices for the added terms, and $n_{Q}$ and $n_{R}$ are the number of rivers and tidal inlets, respectively. Through Eqs. (5a) and (5b), each river and tidal inlet included in the analysis has its own term with its associated exponents, thus allowing for a separation of their respective influence on tidal heights.

The terms in Eqs. (4a) and (4b) can be rewritten for use in a regression into two components, a stage model $s\left(t_{j}\right)$ and a tidal-fluvial model $f\left(t_{j}\right)$; by setting $n_{Q}=n_{R}=1$ in Eqs. (5a) and (5b), we have where the subscripts $s$ and $f$ denote the stage and tidalfluvial models, respectively; the superscripts $c$ and $s$ refer to the cosine and sine terms, respectively; $c_{i}(i=0,2)$ are the model parameters for the stage model; $d_{i, k}(i=0,2)$ are the model parameters for the tidal-fluvial model; and $p_{s}, q_{s}, r_{s}, p_{f}, q_{f}$, and $r_{f}$ are the exponents, determined using an iterative procedure. Iterative regression analyses are run for a representative time series that covers the widest dynamic range in river flow possible, from very low to very high flows, to find the optimal exponents at the analyzed station. Each model is optimized separately, using the original time series of tidal heights for the stage model and a high-passed version of the time series for the tidal-fluvial model. At each iteration of the optimization process, the Rayleigh criterion (defined in the next section) is recalculated based on the exponents of the current iteration and the number of included constituents is adjusted accordingly. An ordinary least squares (OLS) regression is then performed, leading to a new set of coefficients and statistics. The optimization procedure is based on a nonlinear constrained minimization of the residual standard deviation. It uses an interior-point algorithm (Byrd et al. 2000; Waltz et al. 2006), where a sequence of approximate minimization problems is solved using a direct (Newton) step, or a conjugate gradient step if a direct step cannot be taken-for example, when the approximate problem is not locally convex near the current iteration. The procedure is implemented in the MATLAB's fmincon function (MathWorks 2012). Exponents are allowed to vary over the range of $0.2 \leq p \leq 2,1 \leq q \leq 2.5$, and $0.2 \leq$ $r \leq 1.5$, for both the stage and tidal-fluvial models, and initial guesses are set to the theoretical values used by Kukulka and Jay (2003a,b), summarized in Table 1.

Since a different response can be expected from the diurnal, semidiurnal, etc., frequencies, band-specific 
TABLE 1. Selected constituents, optimized exponents, and Rayleigh criteria for the stage and tidal-fluvial models $\left(\mathrm{D}_{1}-\mathrm{D}_{8}\right)$, compared to theoretical exponent values (KJ) from Kukulka and Jay (2003a,b), for an 8-yr-long record (June 2003-June 2011) at Vancouver, WA Results were obtained with two separate discharge terms $\left(n_{Q}=2\right), \eta=0.15$, and mean SNR $\geq 2$ based on a correlated noise model.

\begin{tabular}{|c|c|c|c|c|c|c|c|}
\hline \multirow[b]{3}{*}{ LOR } & \multirow[b]{3}{*}{ Models } & \multirow[b]{3}{*}{ Constituents } & \multicolumn{4}{|c|}{ Exponents } & \multirow{3}{*}{$\begin{array}{c}\text { Rayleigh criteria } \\
\left(\mathrm{h}^{-1}\right)\end{array}$} \\
\hline & & & \multicolumn{2}{|c|}{ Discharge terms } & \multicolumn{2}{|c|}{ Range term } & \\
\hline & & & $Q_{\text {Bon }}^{p_{1}}$ & $Q_{\mathrm{Wil}}^{p_{2}}$ & $R_{\text {Ast }}^{q}$ & $Q_{\text {tot }}^{r}$ & \\
\hline \multirow[t]{2}{*}{$8 \mathrm{yr}$} & Stage, KJ & - & 0.67 & 0.67 & 2.00 & 1.33 & - \\
\hline & $\mathrm{D}_{1}$ to $\mathrm{D}_{8}, \mathrm{KJ}$ & Same as below & 1.00 & 1.00 & 2.00 & 0.50 & $1.089 \times 10^{-3}$ \\
\hline \multirow[t]{9}{*}{$8 \mathrm{yr}$} & Stage & - & 1.39 & 1.07 & 1.04 & 0.37 & - \\
\hline & $\mathrm{D}_{1}$ & $\sigma_{1}, Q_{1}, O_{1}, \mathrm{NO}_{1}, K_{1}, J_{1}, \mathrm{SO}_{1}, v_{1}$ & 1.46 & 0.71 & 2.48 & 0.26 & $9.304 \times 10^{-4}$ \\
\hline & $\mathrm{D}_{2}$ & $\varepsilon_{2}, \mu_{2}, N_{2}, M_{2}, L_{2}, S_{2}, \mathrm{MSN}_{2}$ & 1.20 & 0.86 & 1.30 & 1.03 & $1.006 \times 10^{-3}$ \\
\hline & $\mathrm{D}_{3}$ & $\mathrm{MO}_{3}, \mathrm{MK}_{3}, \mathrm{SK}_{3}$ & 0.53 & 0.48 & 2.38 & 0.53 & $8.402 \times 10^{-4}$ \\
\hline & $\mathrm{D}_{4}$ & $\mathrm{MN}_{4}, M_{4}, \mathrm{SN}_{4}, \mathrm{MS}_{4}, \mathrm{SK}_{4}$ & 0.79 & 0.68 & 2.44 & 0.25 & $9.149 \times 10^{-4}$ \\
\hline & $\mathrm{D}_{5}$ & $2 \mathrm{MK}_{5}, 2 \mathrm{SK}_{5}$ & 0.96 & 0.96 & 2.14 & 0.20 & $1.065 \times 10^{-3}$ \\
\hline & $\mathrm{D}_{6}$ & $2 \mathrm{MN}_{6}, \mathrm{M}_{6}, 2 \mathrm{MS}_{6}, \mathrm{MSK}_{6}$ & 1.00 & 1.00 & 2.00 & 0.50 & $1.089 \times 10^{-3}$ \\
\hline & $\mathrm{D}_{7}$ & $3 \mathrm{MK}_{7}$ & 1.00 & 1.00 & 2.00 & 0.50 & $1.089 \times 10^{-3}$ \\
\hline & $\mathrm{D}_{8}$ & $M_{8}$ & 1.00 & 1.00 & 2.00 & 0.50 & $1.089 \times 10^{-3}$ \\
\hline
\end{tabular}

exponents are calculated by dividing the tidal-fluvial model into submodels, one per frequency band of interest:

$$
f\left(t_{j}\right)=f_{D_{1}}\left(t_{j}\right)+f_{D_{2}}\left(t_{j}\right)+\cdots+f_{D_{n_{f}}}\left(t_{j}\right),
$$

where the $D_{i}$ models $\left(i=1,2, \ldots, n_{f}\right)$ are composed of all frequencies within the diurnal, semidiurnal, etc., frequency bands. This division relies on the assumption that each of the constituents within a given tidal band is influenced by discharge and ocean tidal range in a similar way and can be represented by the same set of exponents. In NS_TIDE, $n_{f}$ is allowed to be as high as 12 , which corresponds to half the sampling frequency (usually $1 \mathrm{~h}$ ) or Nyquist frequency.

The final coefficients $\left(c_{0}, c_{1}, c_{2}, d_{0, k}, d_{1, k}\right.$, and $\left.d_{2, k}\right)$ in Eq. (6) are determined, once the exponents are optimized, by IRLS regression analyses (Huber 1996; Leffler and Jay 2009) to best fit the observations, denoted $y\left(t_{j}\right)$. This takes the form of an overdetermined system composed of all models and submodels (see the appendix), whose solution to the IRLS fit is obtained by minimizing the sum of weighted residuals

$$
E=\sum_{j=1}^{m} w_{j}^{2}\left[h\left(t_{j}\right)-y\left(t_{j}\right)\right]^{2},
$$

where $w$ is a weighting function. NS_TIDE uses the matrix inversion mechanism built into MATLAB's robustfit function (MathWorks 2012). The IRLS naturally de-weighs events that increase residual variance, so that the final coefficients may differ slightly from the coefficients from the last OLS iteration. The level of confidence in the computed parameters is, however, increased, leading to better hindcast under most conditions. Details of the solution are given in the appendix.

When tidal amplitude vanishes, any increase in discharge should leave the tide unchanged (i.e., vanished). The equations, in their current form, may produce artificial, negative amplitudes beyond that point. In fact, the discharge term $d_{1, k} Q^{p_{f}}$ is usually opposed in phase with the constant term $d_{0}$ and thus represents a correction to $d_{0}$. It increases indefinitely as the discharge $Q$ increases, regardless of the value of $d_{0}$. Consequently, when the correction exceeds the value actually corrected, an artificial tide is created that is out of phase with the rest of the signal, thus tainting the hindcast. To reduce this effect, a correction factor based on the total discharge can be applied to the tidal-fluvial model $f\left(t_{j}\right)$ of Eq. (6):

$$
f\left(t_{j}\right)=f\left(t_{j}\right) \times \min \left\{1,\left[Q_{\text {th }} / \sum_{u=1}^{n_{Q}} Q_{u}\left(t_{j}\right)\right]^{\kappa}\right\},
$$

where the threshold discharge value, $Q_{\mathrm{th}}$, and exponent $\kappa$ can either be determined automatically (i.e., included as a parameter to be optimized) or specified by the user for each frequency band. Roughly, this correction switches gradually the tidal-fluvial model off when the threshold discharge is exceeded, such that most of the variations in water levels are accounted for by the stage model alone. It prevents the tidal amplitudes from becoming negative-or the phases to be artificially shifted by $\sim 180^{\circ}$ - as the discharge increases beyond the point where the tide vanishes. This only occurs in extreme cases where the river flow is so strong that the tide is completely extinguished. In less extreme cases (when tides do not disappear), such a correction is not needed. 
If activated, the correction is applied after the Rayleigh criterion is determined and before the IRLS fit as opposed to a postfit correction.

\section{d. Constituent selection and Rayleigh criterion}

Most HA codes use a twofold strategy to select constituents for analysis. The Rayleigh criterion (Godin 1972 ) is used a priori to select constituents to include in the analysis, and the significance of constituents based on error estimates (detailed in next section) is used a posteriori to exclude those that are not significant. The Rayleigh criterion determines a minimal allowable frequency separation between neighboring constituents. Frequencies are selected in accordance with the Rayleigh criterion in a predetermined order following a decision tree based on tidal potential amplitudes (e.g., Foreman 1977). In traditional HA, the Rayleigh criterion states that, given a record composed of $m$ samples evenly spaced by a time step $\Delta t$, only constituents whose frequencies are more than $(m \Delta t)^{-1}$ apart from each other can be resolved. By this definition, the length of record $(\mathrm{LOR}=m \Delta t)$ is the only parameter that determines which constituents are selected, but the Rayleigh criterion may be either too exclusive for low frequencies or too inclusive for higher frequencies (Jay and Flinchem 1999). Such a treatment is valid for stationary signals where only pure spectral lines are considered. In contrast, nonstationarity adds a continuous spectral background that broadens and transforms the tidal spectral lines into cusp-like-shaped peaks that reflect the intensity of modulation of the tides (Munk et al. 1965). As a result, even though the LOR suggests that close frequencies can be resolved, their overlapping cusps lead to erroneous estimates of tidal properties (e.g., Godin 1999). Preliminary tests performed on the implemented model with a LOR-based Rayleigh criterion highlighted this tendency, with the energy of the estimated spectrum greatly exceeding the energy of the original signal because of ill conditioning of the IRLS system.

To obtain a meaningful spectrum, the Rayleigh criterion is redefined in NS_TIDE in such a way that "contaminated" frequencies are eliminated, thereby providing an independent set of constituent basis functions. Furthermore, the constituent selection is performed following a decision tree that is based on the actual order of importance of the constituents rather than on tidal potential amplitude, thus naturally incorporating nonlinear constituents in shallow water. For example, the constituent $M_{3}$, which is the dominant terdiurnal constituent according to tidal potential amplitudes (Foreman 1977), comes after $\mathrm{MO}_{3}$ and $\mathrm{MK}_{3}$ in shallow water in terms of importance. A new decision tree is implemented in NS_TIDE through the computation of a standard HA prior to each nonstationary analysis, to decide on the order of inclusion of the constituents. Thus, the average tidal amplitudes of the analyzed signal in a standard HA are the basis from which constituents are selected.

A new Rayleigh criterion $\Delta \sigma$ is defined, starting from the fact that the width of the cusps formed around the tidal spectral lines is related to the low-frequency spectrum of the nonlinear forcing. For two adjacent tidal frequencies $\sigma_{1}$ and $\sigma_{2}$, the minimal allowable frequency separation $\Delta \sigma$ is given by

$$
\left|\sigma_{1}-\sigma_{2}\right|>\Delta \sigma \quad \text { with } \quad \frac{\int_{0}^{\Delta \sigma} \hat{h}(\sigma) d \sigma}{\int_{0}^{\infty} \hat{h}(\sigma) d \sigma}=1-\eta,
$$

where $\hat{h}(\sigma)$ is the normalized power spectrum of either $Q^{p}$ or $1 / Q^{r}$, and $\eta$ is a user-defined criterion representing a fraction of its total spectral power. The numerator in Eq. (10) represents a region of high power in the lowfrequency band, whose width $\Delta \sigma$ increases as a function of the background modulations. The power spectrum $\hat{h}(\sigma)$ of $Q^{p}$ and $1 / Q^{r}$ typically has maximum amplitude around annual and semiannual periods and then decays with increasing frequency at a rate that is a function of both the exponents $p$ and $r$ and forcing $Q ; \hat{h}(\sigma)$ is calculated separately for each term in Eqs. (5a) and (5b), by letting $R$ be equal to 1 since energy at periods of $\sim 15$ and $\sim 28$ days would otherwise appear in the spectrum $\hat{h}(\sigma)$ due to neap-spring variations in $R$, with the adverse effect of lengthening $\Delta \sigma$. The final value for $\Delta \sigma$ is calculated as the maximum of the LOR-based Rayleigh criterion, $\Delta \sigma_{\mathrm{LOR}}=(m \Delta t)^{-1}$, and all $\Delta \sigma_{i}\left(i=1, n_{Q}+n_{R}\right)$ obtained from Eq. (10) for each term in Eqs. (5a) and (5b): $\Delta \sigma=\max \left(\Delta \sigma_{\mathrm{LOR}}, \Delta \sigma_{i}\right)$.

Since more than one tidal-fluvial submodel (one per tidal band) can be defined through Eq. (7), their associated exponents will lead to band-specific Rayleigh criteria (e.g., Table 1), even if the parameter $\eta$ remains unchanged. This is made possible because $\hat{h}(\sigma)$ in Eq. (10) is not calculated from the subtidal continuum of the input tidal heights but from the low-frequency power of $Q^{p}$ and $1 / Q^{r}$, which varies from one submodel to another because of differences in $p$ and $r$ among the bands. Thus, the more variability in the forcing $Q$ and the higher the exponents $p$ and $r$, the more restrictive the Rayleigh criterion must be. The width of the cusp formed around each dominant frequency is therefore proportional to the width of the low-frequency power associated with its corresponding submodel basis function. Other attempts to establish fixed ratios relating the intensities of major 
constituent cusps to that of the near-zero frequency (e.g., Munk et al. 1965) were not very successful and are hard to generalize from one station to another.

There is a trade-off between the number of constituents included in the analysis and the independence of the terms composing the basis functions. With fewer constituents (smaller $\eta$ ) the overlap between frequencies is less, but the calculated amplitudes will include unknown contributions from nearby unresolved frequencies, and vice versa. For stationary tides, this problem is avoided by inferring unresolvable constituents from resolved constituents using known (constant) amplitude ratios and phase differences. Such a course is not applicable to nonlinear and nonstationary tides by definition. When nonstationary processes occur on short time scales relative to the period needed to separate frequencies, trying to distinguish the satellite peaks by inference (e.g., $P_{1}$ from $K_{1}$ ) is meaningless. Moreover, nonlinear mechanisms transfer energy from the major constituents to adjacent frequencies, making the admittance discontinuous. For example, near $M_{2}, H_{1}$, and $\mathrm{H}_{2}$ have an apparent admittance orders of magnitude greater than that of $M_{2}$, because almost all the energy at $H_{1}$ and $H_{2}$ comes from nonlinear transfer (e.g., Pugh and Vassie 1976). The number of tidal components thus needs to be restricted, through the parameter $\eta$, in order to keep the spectral elements as independent as possible. In NS_TIDE, the user-defined $\eta$ parameter allows the user to tighten the tolerance related to constituent selection. If $\eta$ is set too high, the error calculation (detailed next) will help identify constituents that covary with others, so that they can be excluded a posteriori. Thus, the modified Rayleigh criterion and the error analysis work together to arrive at an analysis with an optimal number of constituents. With strongly nonstationary tidal signals, however, more experimentation with these criteria may be needed than is the case with nearly stationary signals.

For stationary tidal signals, nodal modulations of the main tidal constituents by their satellites are an important consideration. But nodal modulations are, as a rule, small in rivers relative to the effects of stage variations. They are dominated-and any attempt to resolve them is contaminated-by fluvial modulations. In practice, it is virtually impossible to separate the modulation effects on the main tidal constituents by river flow and tidal range from those stemming from changes in lunar declination, even for time series longer than 18.6 years; this follows directly from the redefinition of the Rayleigh criterion. In standard HA, all satellites are usually ignored and amplitudes and phases are determined for all major constituents that can be resolved given the length of the record. Postfit corrections are then applied to account for the presence of the satellites, which modulate the calculated amplitudes and phases. They are defined in terms of a nodal amplitude amplification factor and a nodal angle, which are assumed to vary in the same way as the gravitational potential. This assumption, if invalidated, may lead to systematic errors in the estimation of tidal constituents, as shown by Shaw and Tsimplis (2010). Thus, an underestimation of the equilibrium nodal variation can be associated with nonlinear frictional damping (e.g., Amin 1983, 1985), whereas an overestimation of the equilibrium nodal variation may be due to strong shallow-water interactions (e.g., Amin 1993). Similarly, significant deviations from the equilibrium constants may occur in rivers due to friction and shallow-water effects. Moreover, these relationships are likely to change over time as the river conditions change. Therefore, tidal potential theory is not used in NS_TIDE to dictate relationships between tidal constituents in rivers. Although nodal corrections were not included in our analyses, the time series of tidal range $R$ includes nodal modulations of the dominant diurnal and semidiurnal constituents at the ocean entrance, which are nonlinearly propagated up to the analysis station.

\section{e. Error estimation}

The least squares solution of a HA produces statistical estimates of the constituent properties, whose error variances need to be evaluated in order to determine their significance. Two error models that differ from the models in T_TIDE (see Pawlowicz et al. 2002) were implemented in NS_TIDE, namely, an uncorrelated noise model and a correlated noise model. Each model constructs a covariance matrix of the regression coefficients and generates an ensemble of replicates-assuming a multivariate normal distribution - to compute uncertainty estimates for the stage and tidal-fluvial model parameters in Eq. (6). Each replicate is then converted into constituent parameters using Eqs. (A6)-(A11) and (A13)-(A15), and confidence bounds are estimated for both the regression coefficients and the constituent amplitudes and phases as a function of time. An IRLS solution is used, following Leffler and Jay (2009), to provide robust statistics on the regression coefficients.

In the following development, we define the covariance matrices needed in each of the error models. The error models assume a signal model of the form $\mathbf{h}=\mathbf{A} \mathbf{x}+\boldsymbol{\varepsilon}$, where $\mathbf{h}$ is a vector of the observed values, $\mathbf{A}$ is a matrix of the basis functions evaluated at measurement times, $\mathbf{x}$ is the model parameter vector, and $\boldsymbol{\varepsilon}$ is the measurement noise vector, including nonharmonic signals (i.e., correlated in time). The least squares, 
maximum likelihood, best linear unbiased estimate of $\mathbf{x}$ is given by

$$
\mathbf{x}=\left(\mathbf{A}^{\mathrm{T}} \mathbf{C}_{\boldsymbol{\varepsilon}}^{-1} \mathbf{A}\right)^{-1} \mathbf{A}^{\mathrm{T}} \mathbf{C}_{\boldsymbol{\varepsilon}}^{-1} \mathbf{h},
$$

where $\mathbf{C}_{\varepsilon}$ is the covariance of $\boldsymbol{\varepsilon}$. For the uncorrelated noise model, $\mathbf{C}_{\varepsilon}=\sigma^{2} \mathbf{I}$, where $\mathbf{I}$ is the identity matrix and $\sigma^{2}$ is estimated from the residual as follows:

$$
\sigma^{2}=\frac{1}{N-M}(\mathbf{A x}-\mathbf{h})^{\mathrm{T}}(\mathbf{A} \mathbf{x}-\mathbf{h}),
$$

where $N$ is the number of data samples and $M$ is the number of harmonic coefficients. This yields the covariance matrix of $x$ for uncorrelated noise:

$$
\mathbf{C}_{x u}=\left(\mathbf{A}^{\mathrm{T}} \mathbf{C}_{\varepsilon}^{-1} \mathbf{A}\right)^{-1}=\left(\mathbf{A}^{\mathrm{T}} \mathbf{A}\right)^{-1} \sigma^{2}
$$

For the correlated noise model, we consider the Fourier representation of $\boldsymbol{\varepsilon}=\mathbf{F} \hat{\boldsymbol{\varepsilon}}$ (strictly valid only if the sample interval is constant), where $\mathbf{F}$ is the Fourier matrix of sines and cosines evaluated at the observation times and $\hat{\boldsymbol{\varepsilon}}$ is a vector of Fourier components. Thus, via the Fourier transform, $\hat{\boldsymbol{\varepsilon}}$ and $\boldsymbol{\varepsilon}$ are related by $\hat{\boldsymbol{\varepsilon}}=\mathbf{F}^{-1} \boldsymbol{\varepsilon}$. The covariance of $\boldsymbol{\varepsilon}$ is given by $\mathbf{C}_{\varepsilon}=\mathbf{F} \mathbf{C}_{\hat{\varepsilon}} \mathbf{F}^{\mathrm{T}}$, where $\mathbf{C}_{\hat{\varepsilon}}$ is assumed diagonal (i.e., each Fourier component of $\boldsymbol{\varepsilon}$ is uncorrelated) and is estimated from the power spectrum of residuals. A Daniell average (Daniell 1946) over the Rayleigh bandwidth is used to achieve a stable estimate. The covariance matrix of $x$ for correlated noise is thus given by

$$
\mathbf{C}_{x c}=\left[\left(\mathbf{F}^{-1} \mathbf{A}\right)^{\mathrm{T}} \mathbf{C}_{\hat{\varepsilon}}^{-1} \mathbf{F}^{-1} \mathbf{A}\right]^{-1} .
$$

For evenly spaced data, the error estimate is essentially the same as the correlated noise model in T_TIDE, except for the different bandwidth used to estimate the nontidal noise. The singular value decomposition of $\mathbf{C}_{x c}$ could be computed to analyze the stability and conditioning of the nonharmonic analysis, similar to the approach of Foreman et al. (2009), although we have not pursued this.

Including the weighting matrix $\mathbf{W}$ of the IRLS yields the following covariance matrices for the uncorrelated and correlated noise models, respectively:

$$
\begin{aligned}
& \mathbf{C}_{x u}=\left(\mathbf{A}^{\mathrm{T}} \mathbf{W} \mathbf{A}\right)^{-1} \sigma^{2} \\
& \mathbf{C}_{x c}=\left[\left(\mathbf{F}^{-1} \mathbf{W}^{1 / 2} \mathbf{A}\right)^{\mathrm{T}} \mathbf{C}_{\hat{\varepsilon}}^{-1} \mathbf{F}^{-1} \mathbf{W}^{1 / 2} \mathbf{A}\right]^{-1} .
\end{aligned}
$$

Since the amplitude and phase errors are functions of time in NS_TIDE, the significance of the constituents is assessed based on a time-averaged signal-to-noise ratio (SNR), which is the square of the ratio of tidal amplitude to amplitude error. Constituents whose mean SNR is below the SNR lower limit are excluded from the analysis. The solution is recalculated using the significant constituents only.

\section{Application to river tides}

\section{a. Setting: The lower Columbia River}

The Columbia River enters the northeast Pacific Ocean $30 \mathrm{~km}$ seaward of the Tongue Point tide gauge at Astoria, Oregon (Fig. 1). It is the third largest river in the United States, with an annual average discharge of $\sim 7300 \mathrm{~m}^{3} \mathrm{~s}^{-1}$ and a watershed of $\sim 660500 \mathrm{~km}^{2}$ (Bottom et al. 2005). The lower Columbia River (LCR) spans 235 river kilometers ( $\mathrm{rkm}$ ) from the mouth to the Bonneville Dam, located at the natural head of the tide. The maximum salinity intrusion length is $5-50 \mathrm{~km}$, depending on the river flow and tides. Tidal amplitudes may vary by an order of magnitude due to the annual river flow cycle, and the propagation time from the entrance to rkm 170 almost doubles during high flow periods. Thus, the LCR is a good natural laboratory for the analysis of nonstationary tides.

The Willamette River is the largest tributary to the LCR and enters the river main stem at Portland, $160 \mathrm{rkm}$ from the ocean. Its average discharge of $950 \mathrm{~m}^{3} \mathrm{~s}^{-1}$ accounts for $>40 \%$ of the average flow entering the main stem below Bonneville Dam.

\section{b. Harmonic analysis properties}

NS_TIDE was applied to hourly water level data for an 8-yr period (June 2003-June 2011) from Vancouver, Washington (rkm 172), the second most landward of 10 tide gauges in the LCR (Fig. 1). These data were used here because they illustrate very well the difficult characteristics of river tides. The tides at Vancouver (Fig. 2) exhibit semidiurnal and diurnal tidal variations and have a range that varies from a few centimeters during high flow periods to $\sim 1 \mathrm{~m}$ during fall spring tides. MWL varies by $3-8 \mathrm{~m}$ seasonally with river flow and by $\sim 0.5 \mathrm{~m}$ over stronger neap-spring cycles in fall. The average frequency content of the tidal signal at Vancouver, obtained from a standard HA for the 8-yr time series, contains 63 significant constituents. The admittance varies radically across the diurnal and semidiurnal bands due to nonlinear effects and daily cycles in hydropower demand at Bonneville Dam. Figure 2 shows forcing data used to test NS_TIDE, which include hourly flow in the Columbia River at Bonneville Dam, daily flow in the Willamette River at Portland, and greater diurnal tidal range at Astoria-Tongue Point (rkm 29).

Hourly discharge data at the Bonneville Dam were low passed using a 3-day smoothing window, similar to Godin's (1972) tidal eliminator, in order to remove daily 


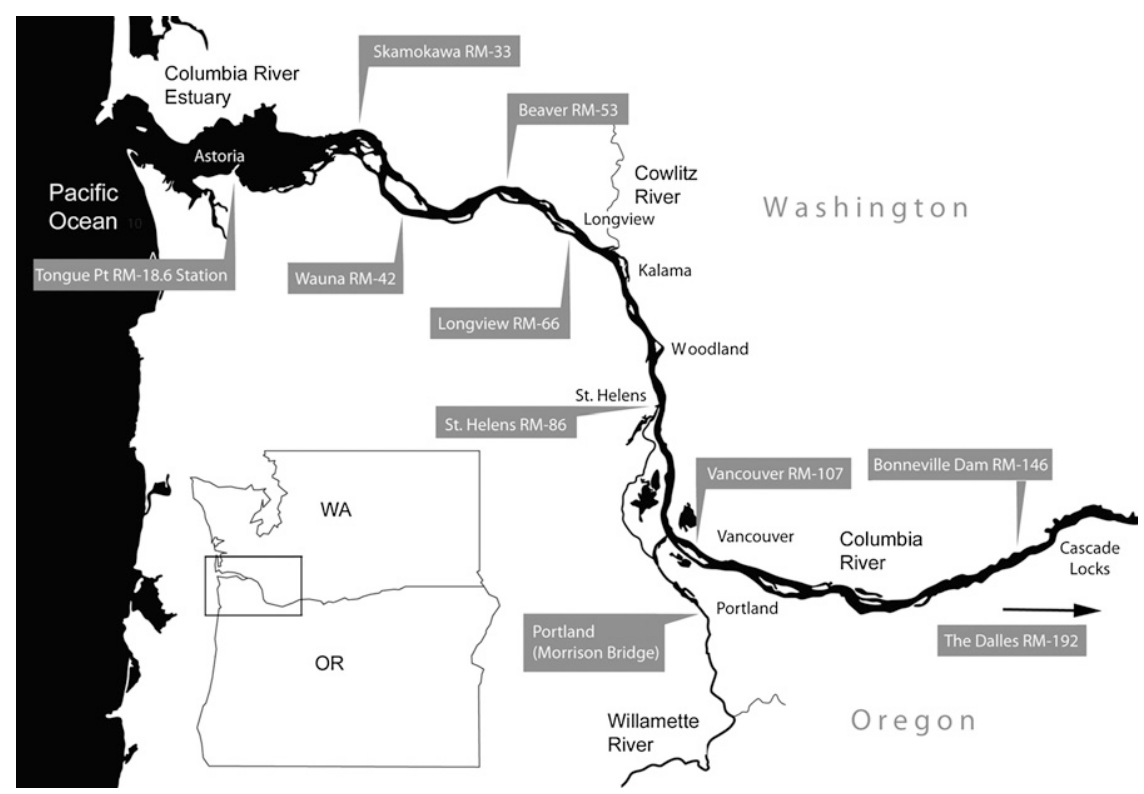

FIG. 1. Map showing tide stations in the LCR, with official river-mile designations; map courtesy of the Port of Portland.

fluctuations caused by hydroelectric operations ("power peaking"). Although our method does not require data prefiltering of forcing data, this was done to eliminate undesirable oscillations in the modeled signal associated with irregular discharge waves at Bonneville Dam. However, the diurnal constituents at Vancouver are still affected by these fluctuations in river flow, propagating from the dam seaward. Fluctuations with periods of 3.5
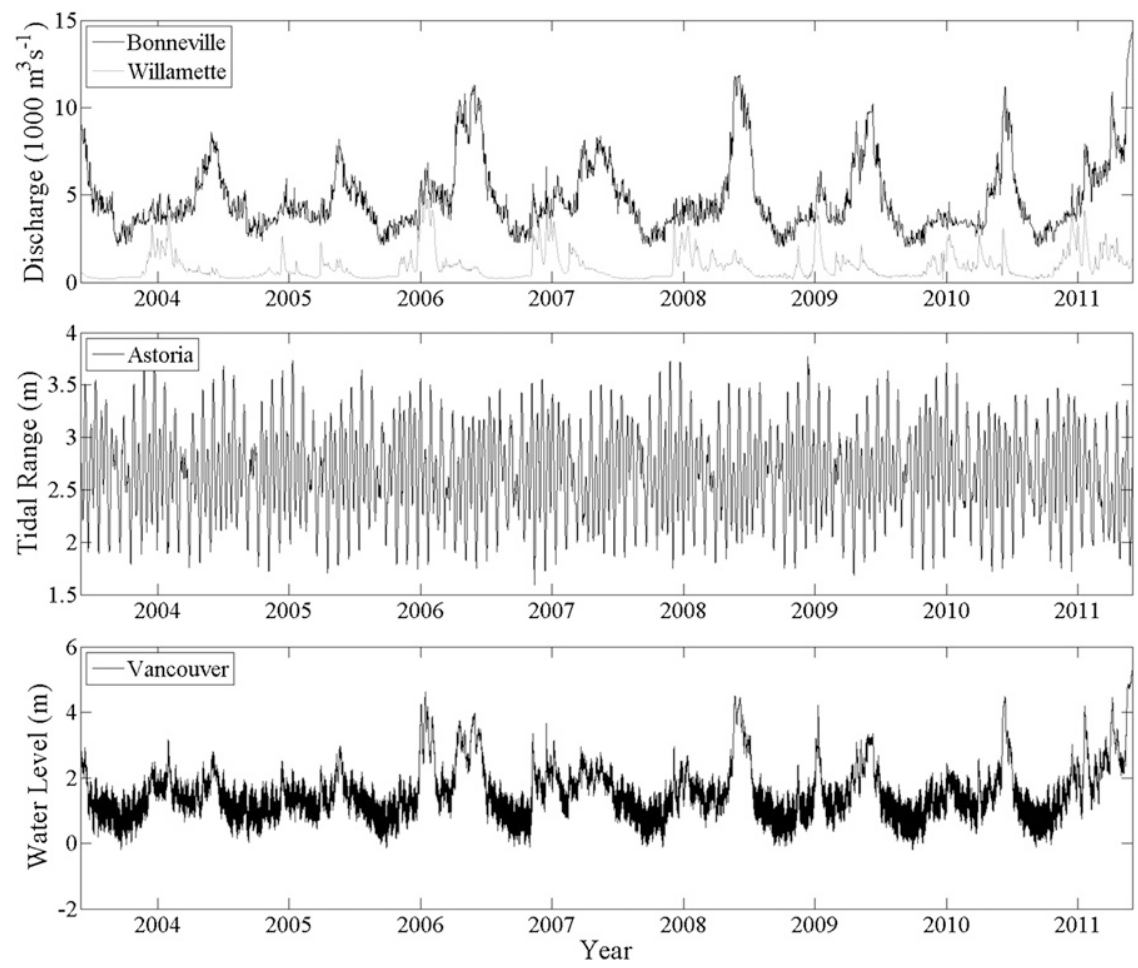

FIG. 2. Forcing variables and tidal response in the LCR from June 2003 to June 2011: (top) discharge forcing at the Bonneville Dam and in the Willamette River, (middle) ocean tidal range forcing at Astoria, and (bottom) tidal response at Vancouver. 
and 7 days related to power peaking also remain in the filtered Bonneville time series and will be propagated to the analysis station. No filtering was applied to the daily discharge data of the Willamette River.

Greater diurnal tidal range was extracted from hourly data at Astoria-Tongue Point. We chose Astoria-Tongue Point at rkm 29 as the reference station for the ocean tidal forcing, because tides at this location are only weakly influenced by river flow and it is the most seaward station for which sufficient data are available. To determine extrema, the hourly water level was high passed and then spline interpolated to 6-min intervals. Maximum and minimum heights were retrieved using a $27-\mathrm{h}$ moving window with 1-h steps. Tidal ranges were calculated from the difference between the maximum and minimum values, and smoothed using a 27 -h moving average to eliminate discontinuities due to steps in the tidal ranges.

The stage and tidal-fluvial models applied to the LCR have the form shown in Eq. (6), modified per Eq. (5a) to include separately the river flows at Bonneville Dam and from the Willamette River (i.e., $n_{Q}=2, n_{R}=1$ ). While including the two discharges separately adds regression parameters and therefore increases error bounds, this adverse effect is compensated by a more accurate representation of the distinct effects of both rivers on tidal heights, especially marked during high flows (see Fig. 2). In fact, high flows are usually not synchronized in the Columbia and Willamette Rivers, and they affect the tide at Vancouver with different lags. Also, the Willamette River flow affects tidal dissipation in the LCR and may reverse in the main stem beyond Vancouver, thus impacting Vancouver water levels (Jay et al. 2011). Sensitivity of the results to this separation was tested for the cases 1) without the Willamette River, 2) with the Willamette and Bonneville Dam flows summed and added as a single discharge term (i.e., $n_{Q}=1$ ), and 3 ) with the Willamette River included as a separate term (i.e., $n_{Q}=2$ ). Sensitivity of MWL, constituent amplitudes and phases, and tidal range to the exponent values and the LOR was also assessed. The discharge threshold $Q_{\text {th }}$ and exponent $\kappa$ in Eq. (9) were set to $11000 \mathrm{~m}^{3} \mathrm{~s}^{-1}$ and 10, respectively, with the effect of switching the tidal-fluvial model off for discharges exceeding this limit. Since there were very few high-discharge/low-tide events during the 8-yr time series analyzed at Vancouver, these parameters were optimized using shorter analysis windows centered on these events. The final $Q_{\text {th }}$ and $\kappa$ values were chosen based on the optimization results and visual inspection of the tidal record; they are applied to all the analyses.

The tidal-fluvial model $f\left(t_{j}\right)$ was divided according to Eq. (7) into eight $D_{i}$ submodels $(i=1,8)$, each having a different set of exponents. The parameter $\eta$ for the
Rayleigh criterion was set to 0.15 , allowing for the resolution of a reasonable number of constituents while keeping the error bounds relatively low, given the nonstationarity of the analyzed signal. Values of 0.05 and 0.5 were also tested to assess the sensitivity of the model to the parameter $\eta$. The significance of the constituents was determined using a correlated noise model with a mean $\mathrm{SNR} \geq 2$; a mean SNR $\geq 10$ was also tested for comparison purposes, as well as an uncorrelated noise model. The number of realizations for the error estimation was set to 300 . A Cauchy IRLS function with a tuning constant of 2.385 was used.

\section{c. Results}

\section{1) MODEL PARAMETERS}

Table 1 summarizes the derived model exponents and compares them to the theoretical values of Kukulka and Jay (2003a,b). Departure of the exponents from theoretical values are observed for the stage and $D_{1}-D_{5}$ models, while the $\mathrm{D}_{6}-\mathrm{D}_{8}$ models present the same values as in Eq. (3), meaning that the optimization algorithm does not find the error to be decreasing in any direction. Deviations are partly explained by the linear form of the present model, as opposed to the logarithmic form of Kukulka and Jay's models. Differences from theoretical values also arise from the highly nonlinear character of the tides at Vancouver and the fact that channel geometry (e.g., flow-variable width) affects tides in a frequency-dependent manner, leading to bandspecific exponents. The differences in the exponents of the two discharge terms are quite significant for the stage, and $D_{1}$ and $D_{2}$ models. Their inclusion as two separate terms is therefore essential in order to adequately capture their respective effects on MWL and tidal properties.

Also shown in Table 1 are the selected constituents for each tidal-fluvial submodel $\left(\mathrm{D}_{1}-\mathrm{D}_{8}\right)$, along with their respective Rayleigh criterion. A total of 31 constituents are included in the analysis, compared to 63 with a LORbased Rayleigh criterion of $1.422 \times 10^{-5}$. This reduction is attributable to the redefined Rayleigh criterion. For example, $P_{1}$-the third largest diurnal constituent in terms of tidal potential amplitudes-cannot be separated from $K_{1}$ with an 8-yr analysis because of the nonstationarity of the signal. In fact, in highly nonstationary environments, the LOR does not dictate the frequency separation between neighboring constituents anymore, as shown by Eq. (10). Another feature related to the redefinition of the Rayleigh criterion is its associated decision tree, based on the local importance of constituents as opposed to the order of inclusion presented by Foreman (1977). This explains, for example, why $M_{3}$ does not appear in the $\mathrm{D}_{3}$ model. 
(a)
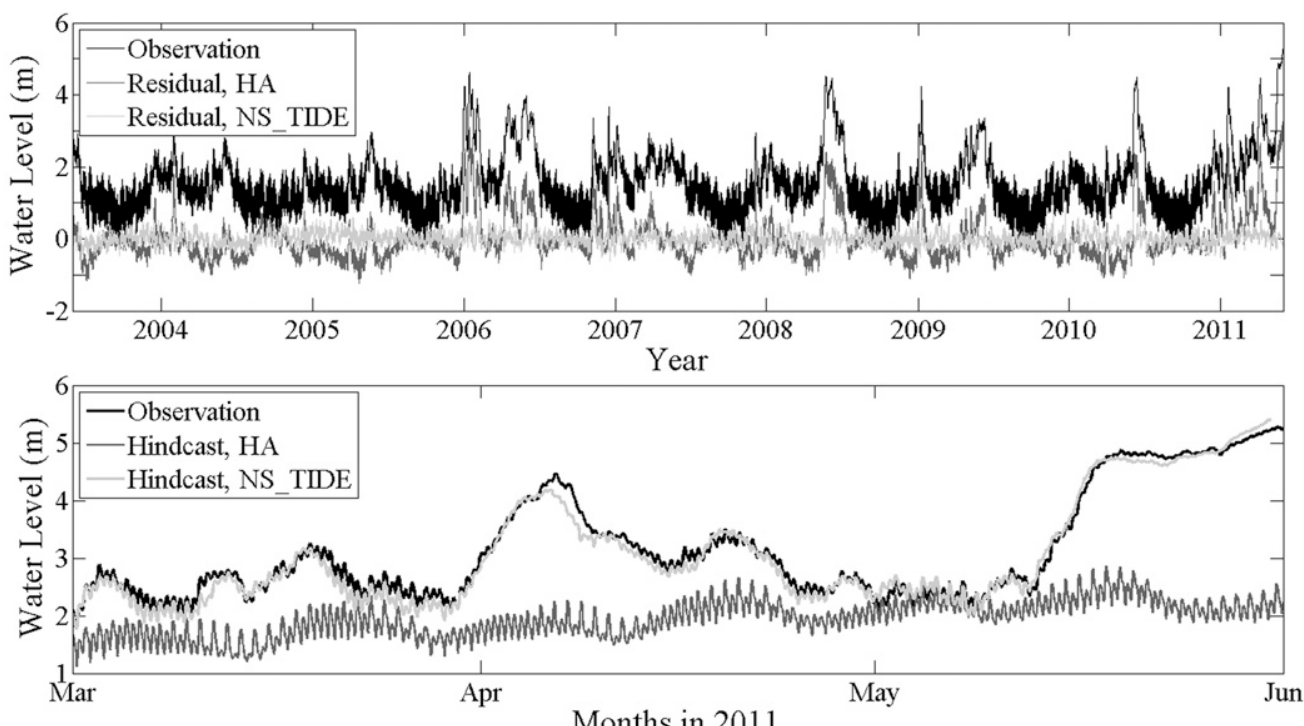

(b)
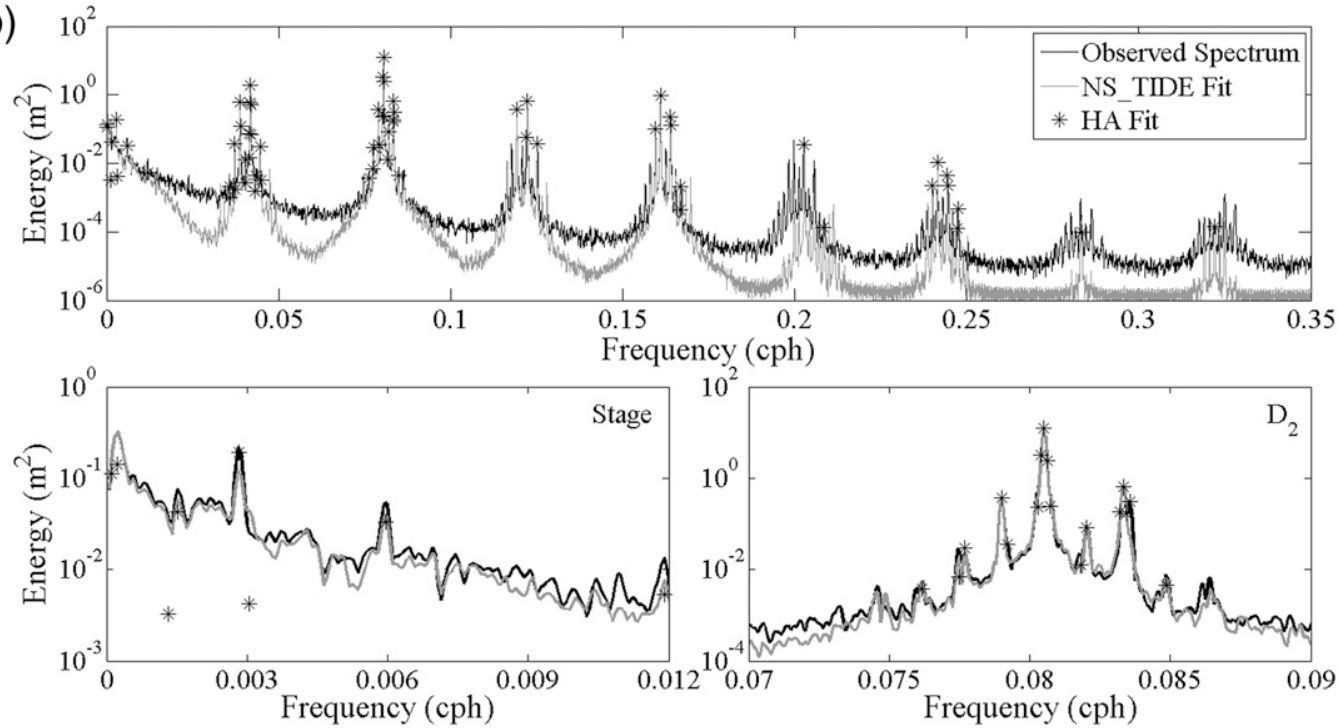

FIG. 3. Results for standard HA and NS_TIDE at Vancouver, WA, from June 2003 to June 2011. (a) (top) Residuals and (bottom) hindcasts are shown for both methods, for the complete time series and a three-month period in 2011, respectively. (b) (top) Power spectra of the observed signal and IRLS fit for both methods. (bottom) Zoom-ins of the power spectra for the low-frequency (stage) and semidiurnal $\left(\mathrm{D}_{2}\right)$ bands.

\section{2) MODEL PERFORMANCE AND SENSITIVITY}

Shown in Figs. 3a and $3 \mathrm{~b}$ is a comparison of results obtained from standard HA and NS_TIDE at Vancouver, for the analysis period extending from June 2003 to June 2011. Two constituents with periods of 3.5 and 7 days were added in the standard HA to account for lowfrequency fluctuations associated with power peaking at Bonneville Dam. For classical HA, the hindcast explains $38.5 \%$ of the original signal variance and has a rootmean-square error (RMSE) of $0.604 \mathrm{~m}$ and a maximum absolute error of $3.26 \mathrm{~m}$ for tidal heights, as evidenced by the HA residual (Fig. 3a). These results clearly show the inability of HA to resolve the nontidal fluctuations associated with changing river flow. Results for NS_TIDE sharply contrast with those for HA: the hindcast explains $96.4 \%$ of the original signal variance and has an RMSE of $0.165 \mathrm{~m}$ and a maximum absolute error of $0.99 \mathrm{~m}$. Furthermore, the NS_TIDE hindcast shown in Fig. 3a not only follows the low-frequency stage fluctuations of the MWL but exhibits reductions in tidal amplitudes during very high flow periods, unlike 
HA. This performance was achieved with half the constituents used in traditional HA because of more restrictive Rayleigh criteria, but with a larger number of parameters. The latter is 127 for the HA, which corresponds to $2 n+1$, with $n$ equal to the number of constituents, plus the mean sea level. For NS_TIDE, the number of parameters is 288 , which is $2 n_{Q}+3 n_{R}+1$ for the stage model and $2 n\left(n_{Q}+n_{R}+1\right)+n_{f}\left(n_{Q}+2 n_{R}\right)$ for the tidal-fluvial model. While it is vital to recognize the limits on the amount of information in a signal (related to the LOR, number of data, and noise level), the larger number of parameters considerably increases the explained variance and significantly reduces the residual error, compared to standard HA. Because the additional parameters are related to the actual dynamics of river tides, their use also increases the ability of NS_TIDE to predict tides outside of the analysis period.

In Fig. 3b, the IRLS fits obtained from both HA and NS_TIDE are compared to the observed spectrum. The NS_TIDE fit encompasses more of the observed signal energy than classical HA, especially at low frequencies (bottom-left panel), where the harmonics from HA present the largest deviations from the observed spectrum. Because traditional HA assumes low-frequency oscillations to be perfectly sinusoidal and consistent from year to year, the subtidal forecasts computed with HA programs is generally found to be unsatisfactory. In fact, the usual harmonic apparatus, which provides semimonthly (Mf, MSf), monthly (Mm, MSm), semiannual (Ssa), and annual (Sa) constituents, is unable to adequately represent low-frequency river motions that are dominated by tidal-fluvial interactions and nonlinearly generated low-frequency compound tides (Parker 2007). In contrast, the low-frequency spectrum from NS_TIDE shows energy at annual and semiannual periods due to seasonal cycles in discharge, at monthly and semimonthly periods due to the influence of the tidal range term, and at periods of 3.5 and 7 days due to power-peaking fluctuations appearing in the Bonneville discharge term. In the semidiurnal band (bottom-right panel), the NS_TIDE fit almost perfectly coincides with the observed spectrum and reproduces the cusplike shapes formed around the dominant frequencies with great accuracy. Many constituents appearing in the HA fit (e.g., $H_{1}$ and $H_{2}$, which differ from $M_{2}$ by \pm 1 cpy) are far larger than expected from astronomical forcing, because they represent the modulation of major constituents (e.g., $M_{2}$ ) by the flow. In the fluvial context, astronomical $H_{1}$ and $H_{2}$-as well as other frequencies of the HA fit separated by only a few cycles per year from major constituents-are insignificant, and these constituents are not resolved in NS_TIDE because river flow is included directly in the basis functions. Similar observations can be made for the other tidal bands (not shown).

The preceding results are summarized in Table 2 and compared to a series of tests aimed at assessing the sensitivity of the model. First presented is the performance obtained when Kukulka and Jay's (2003a,b) exponents are used for the stage and tidal-fluvial models, in place of the optimized exponents shown in Table 1. Results compare well with the reference analysis, with an explained variance $\geq 90 \%$. The RMSE is increased by about $3 \mathrm{~cm}$, mainly due to differences in MWL during very high flow conditions. Differences in tidal amplitudes and phases are minor and their variations as a function of the forcing conditions are well reproduced. The low sensitivity of model results to the exponents is partly because variations in exponents are compensated by changes in model coefficients in a way that the resulting time series remain almost unaffected.

Two 1-year analyses were also performed for a year with very strong river flow variations-June 2010 to June 2011-and a year characterized by much lower flows-June 2004 to June 2005 (Fig. 2). Results show similar performances to the reference hindcast despite the smaller number of data points used, because the parameters are tuned to the conditions prevailing during each period of analysis. However, predictions based on the low flow year analysis are likely to be inaccurate if used under conditions that fall outside the range within which the model parameters were determined. This is shown in Table 2 through predictions made for the June 2003 to June 2011 period using the parameters from the high and low flow year hindcasts. The analysis period must therefore cover most conditions encountered in the system in order for the tidal properties to be predicted with precision. In some cases, this can only be achieved using longer analysis windows or periods of greater range of flow conditions.

Model sensitivity to constituent selection and rejection was assessed through variations of the $\eta$ parameter of the Rayleigh criterion and the tolerance on the mean SNR for the error model. By varying $\eta$ from 0.05 to 0.5 , the number of included constituents increases and, by extension, the number of parameters. Reducing the number of constituents only slightly degrades model performance. Similarly, the inclusion of more constituents does not significantly improve the results. On one extreme, if only one constituent per tidal band is included, then the analysis is similar to CWT, where all the energy contained in a tidal band is carried by a single frequency, with no distinction made between constituents. The resulting fit would explain most of the variance but with insufficient energy at minor tidal frequencies. On the other extreme, if the LOR is the only 


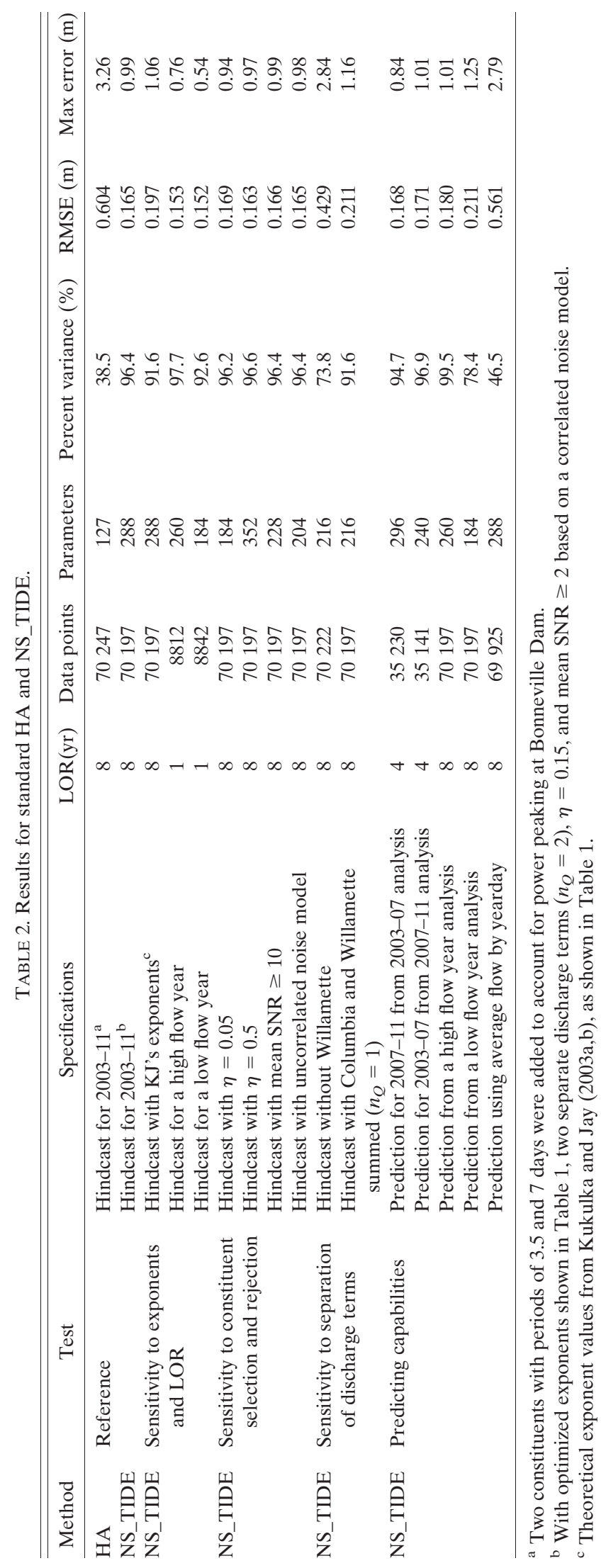




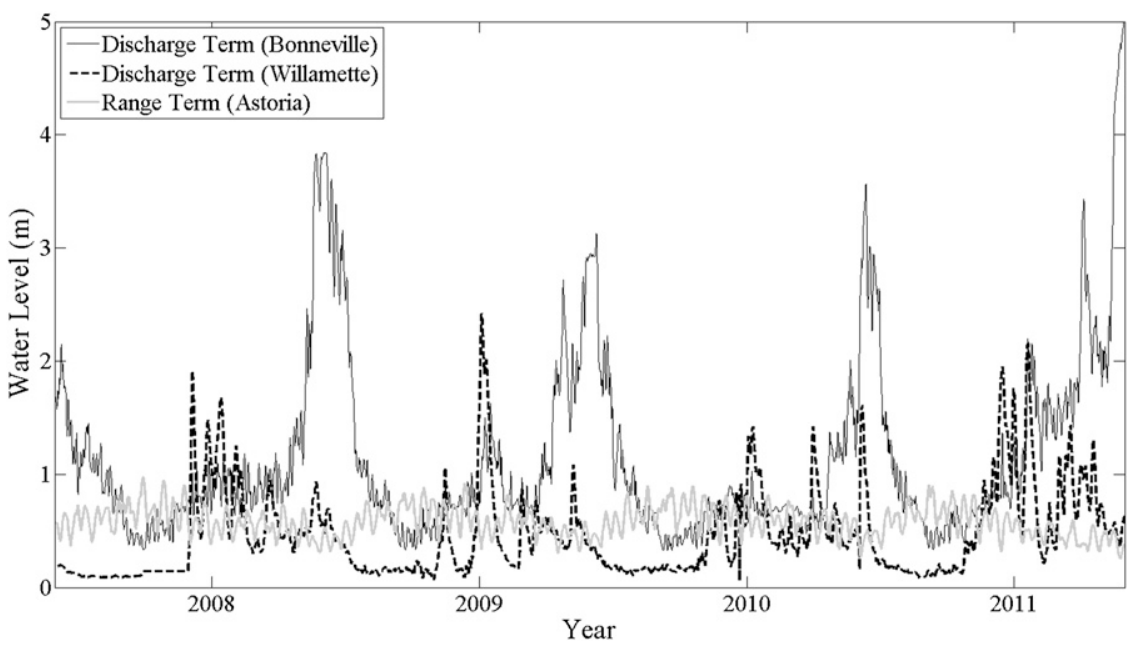

FIG. 4. Individual contributions of each term of the stage model to the MWLs at Vancouver, WA, from June 2007 to June 2011, representing the discharge at Bonneville Dam, the discharge in the Willamette River, and the tidal range at Astoria, respectively.

criterion controlling constituent selection, then the included constituents are more dependent, and phase shifts appear between constituents of a given tidal band that represent energy cancellation at nearby frequencies. The resulting linkage between constituents becomes more apparent as the nonlinear interactions between tidal components increase. Although the resulting fit is good, the properties of some minor constituents are not physical but rather an artifact of the analysis method, with correspondingly negative effects on the ability of the results to predict tides outside the analysis period. A moderate $\eta$ value combined with a sufficiently high SNR tolerance lead to a selection that conforms to the local dynamics. Increasing the mean SNR lower limit to 10 actually led to the rejection of seven constituents without affecting model performance. The use of an uncorrelated noise model, instead of the correlated noise model, also led to the rejection of a higher number of constituents, mainly those composing the $\mathrm{D}_{6}-\mathrm{D}_{8}$ tidal bands.

As stressed above, the inclusion of the Willamette River as a separate discharge term is a crucial element in modeling the tides at Vancouver. This is reflected in Table 2, first in a hindcast made without the Willamette River, and second in a hindcast where the same exponents are attributed to both discharge terms by combining them into a single term. Model performance is greatly affected by the exclusion of the Willamette River contribution. The effect of summing both discharges is lesser, but significant, and compares to the results obtained with the Kukulka and Jay's (2003a,b) exponents.

Also presented in Table 2 are the prediction performances obtained with NS_TIDE by dividing the 8-yr period into two equal halves and using the analysis of each half to predict tides for the other. For both predictions, the explained variances are around $95 \%$ or higher, and the RMSE and maximum absolute errors are very close to the 8 -yr reference hindcast. This is because both analysis windows encompass essentially the entire range of present flow conditions. An analysis performed using less dynamic years would likely provide poorer predictions. This is illustrated in Table 2 through two 8-yr predictions made using parameters from a high and low flow year hindcast, respectively, the latter presenting poorer performances during periods of high flows due to an inadequate choice of the reference analysis window. The last entry of Table 2 shows results of a prediction made using the coefficients of the reference hindcast and a discharge time series based on an average river flow by yearday, which, for some systems, is the only available forecast for discharge. Results are better than for HA, but errors remain large because of the large interannual variability of the river flow cycle.

\section{3) Stage Model}

Low-frequency fluctuations at Vancouver are represented by the stage model $s\left(t_{j}\right)$. Figure 4 presents reconstructed time series for each term of the stage model. Results show that the MWL is mostly influenced by the discharge from Bonneville Dam, which follows sharp seasonal cycles with maximum flows usually occurring between March and June. Differences in MWL between high and low flow periods can be as high as $5 \mathrm{~m}$ during high flow years (e.g., 2011). The Willamette River flow, in turn, is much lower on average than the Columbia 


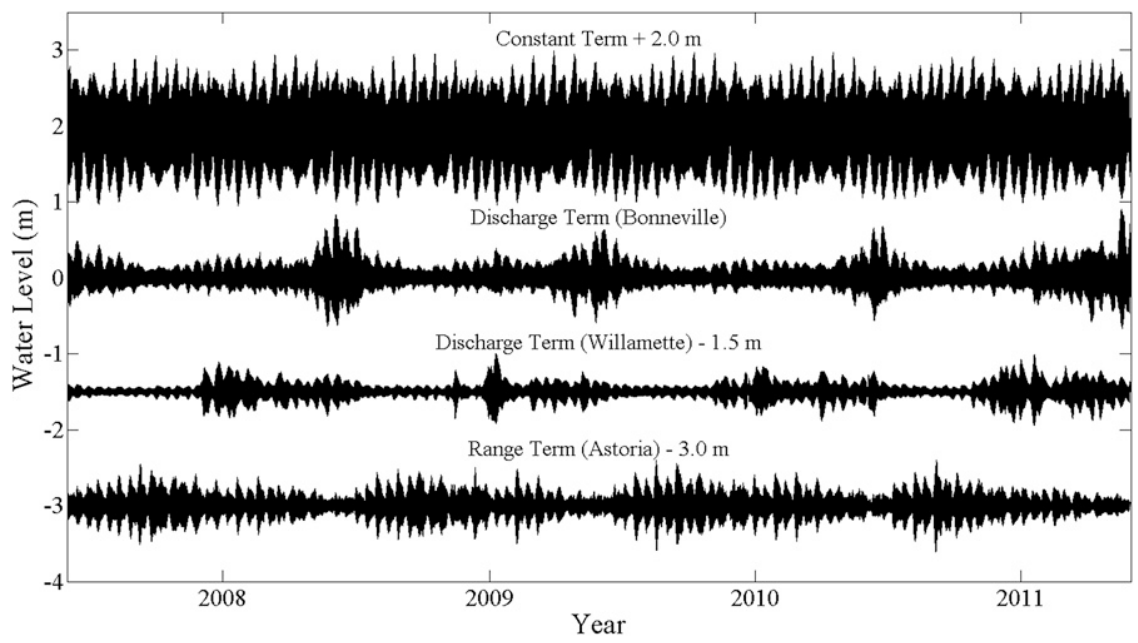

FIG. 5. Individual contributions of each term of the tidal-fluvial model to the tidal heights at Vancouver, WA, from June 2007 to June 2011, representing the astronomical forcing (constant term), the discharge at Bonneville Dam, the discharge in the Willamette River, and the tidal range at Astoria, respectively. Time series are shifted vertically for clarity.

River flow, but it often exhibits high peaks during the winter. Changes in MWL, illustrated in Fig. 4, due to high flows in the Willamette River exceed $2.5 \mathrm{~m}$ during the analysis period. Inclusion of the influence of the Willamette River flow as a separate term is therefore justified, given its marked effect on MWL and its asynchronicity with the Columbia River flow. The range term of the stage model represents the influence of the neap-spring cycle on MWL. Semimonthly oscillations appear in the reconstructed signal shown in Fig. 4, typical of what is usually observed at upstream stations in tidal rivers. These oscillations increase in importance as the flow decreases. As for the constant term $c_{0}$ in $s\left(t_{j}\right)$ (not shown), it gives the vertical shift that best fits the data, which is $-0.61 \mathrm{~m}$ in this case. This represents the difference between the average MWL and the gauge datum, which is set at an estimate of historic extreme low water.

\section{4) TIDAL-FLuVial MODEL}

Figure 5 shows the contribution of each term of the tidal-fluvial model $f\left(t_{j}\right)$ to the total tidal variance, representing the astronomical forcing (constant term), the discharge at Bonneville Dam, the discharge in the Willamette River, and the tidal range at Astoria, respectively. The sum of all terms yields the high-passed time series of predicted tidal heights. To better understand how tidal heights are dynamically connected to each of these terms, we can look at the amplitudes and phases of the constituents that compose the signal. Figure 6 shows the amplitude variations of each separate term as a function of time for the $M_{2}$ tide alone. Their respective phases are $89.1^{\circ}, 257.0^{\circ}, 256.6^{\circ}$, and $4.1^{\circ}$.
Properties of the constant term are an indicator of the amplitude and phase that the $M_{2}$ constituent would reach in the absence of river flow. The phases of both discharge terms differ by about $168^{\circ}$ from that of the constant term, meaning that, to the lowest order, flow reduces tidal amplitudes. In other words, the timedependent amplitudes of the discharge terms are essentially subtracted from the constant term. Since the phase difference is not exactly $180^{\circ}$, the phase of the resulting $M_{2}$ tide will gradually tend toward that of the discharge terms as their amplitude increases. This is consistent with tidal dynamics, as we expect the propagation times to increase as river flow increases. The tidal range term, in turn, makes the constituent properties (both amplitudes and phases) oscillate in accordance with the neapspring cycle.

The resulting amplitudes and phases as a function of time, all terms combined, are shown in Fig. 7, for the $K_{1}$ and $M_{2}$ tides. Traditional HA gives, for $K_{1}$ and $M_{2}$, average amplitudes of 0.13 and $0.22 \mathrm{~m}$ and average phases of $9.3^{\circ}$ and $60.4^{\circ}$, respectively. Results from NS_TIDE show fluctuations around these mean values following the variations in forcing. Amplitudes are significantly reduced and phases shifted as the river flow increases, and both constituent properties show fluctuations that follow the neap-spring cycle, as they react to its associated fortnightly variations in friction.

Amplitude and phase errors, shown as dotted lines in Fig. 7, increase during highly nonstationary events, as the signal becomes more nonlinear. Large errors during very high flows $\left(Q>Q_{\text {th }}\right)$ are, however, reduced by use of Eq. (9). In NS_TIDE, error bounds also increase as more constituents are included in the analysis, since they 


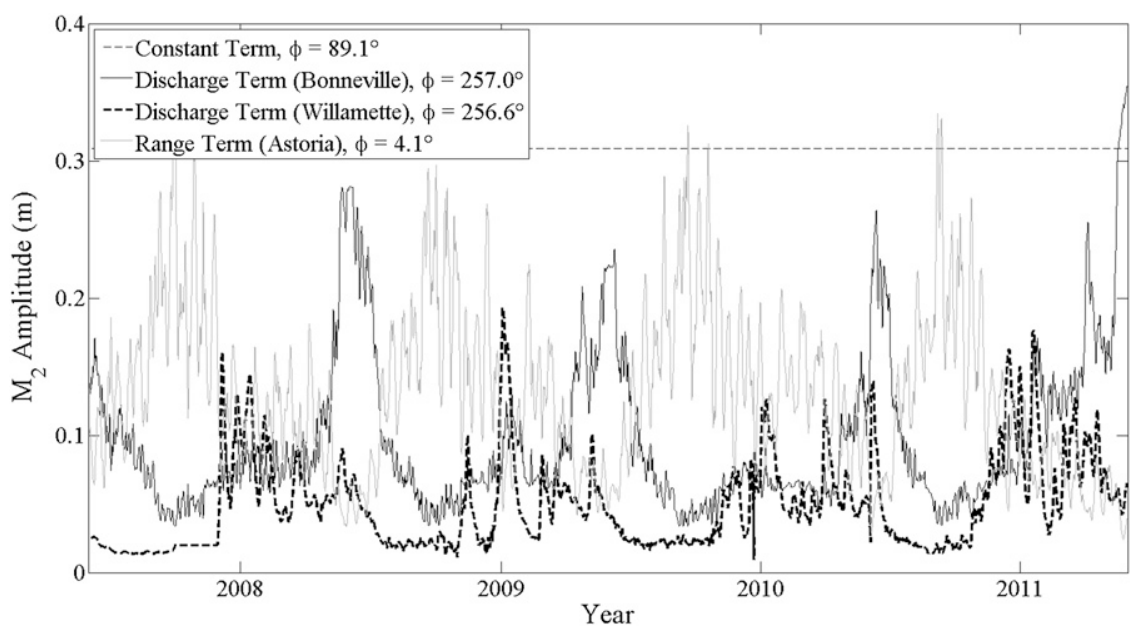

FIG. 6. Individual contributions of each term of the tidal fluvial-model to the $M_{2}$ amplitudes at Vancouver, WA, from June 2007 to June 2011, representing the astronomical forcing (constant term), the discharge at Bonneville Dam, the discharge in the Willamette River, and the tidal range at Astoria, respectively. Phases are shown in the legend.

become more dependent on each other. Applying a more restrictive Rayleigh criterion, therefore, improves the confidence limits and decreases the dependence among constituents, but it determines fewer of them.

\section{Discussion and conclusions}

\section{a. Improvements over conventional tidal analysis methods}

Conventional tidal analysis methods are usually unable to represent the nonstationary nature of river tides, because they are either limited in their time or frequency representation of the phenomenon. Traditional HA gives average amplitudes and phases for a wide range of constituents, but with no information in the time domain. Time series obtained from STHA, using a relatively short moving window compared to the analysis period, typically exhibit increased tidal damping during periods of high discharge accompanied by phase shifts due to increased propagation times of the tidal wave. The effects of tidal range are, however, smoothed out with window lengths that exceed the duration of the neap-spring cycle. In addition, each individual analysis can only be used to predict tides under the exact same flow and tide conditions, which makes

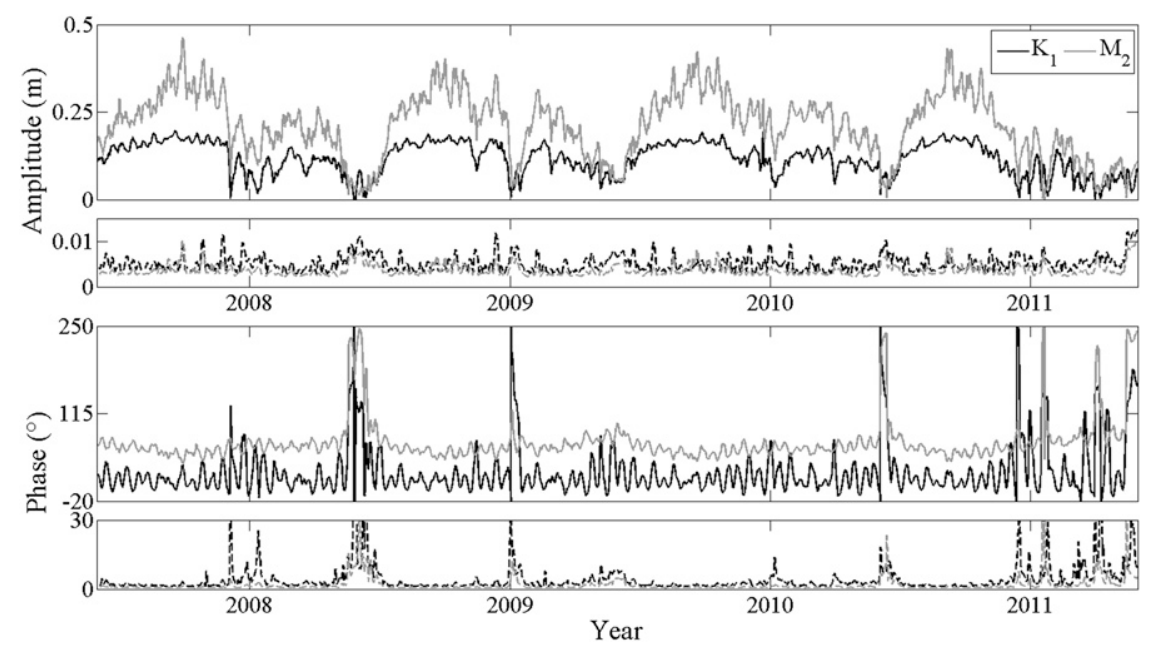

FIG. 7. $K_{1}$ and $M_{2}$ amplitudes and phases (solid lines) at Vancouver, WA, from June 2007 to June 2011, along with their error bounds (dotted lines), based on a correlated noise model. 
STHA useless for prediction. Interpretation of results from STHA is also laborious, because there is no easy way to separate the influence of discharge from different rivers or tidal range on the resulting tide. CWT, in turn, proved to be very successful in the study of nonstationary time series as it provides a good localization of events in time and is invertible (Jay and Flinchem 1995). It nonetheless lacks frequency resolution within tidal bands and is sensitive to sidelobe effects near the ends of the time series.

In contrast, NS_TIDE gives, with a single analysis, time series of amplitudes and phases for each resolved constituent with the same time resolution as the original signal. It is able to solve for more than one frequency per tidal band-although fewer than with standard HAunder highly nonstationary tidal conditions without losing information in the time domain. Moreover, this is done in such a way that the respective contribution of flow and tidal forcing can be quantified and used for hindcast or prediction purposes, if flow predictions are available. Thus, NS_TIDE is able to facilitate dynamical inquiries regarding the evolution of tides as a function of the changing forcing conditions.

\section{b. Merits of the approach compared to dynamic circulation models}

The presented method provides a pointwise (0D) description of the tides and MWL that can potentially be extended to a 1D description by spatial interpolation of the model parameters. In comparison, dynamic circulation models $(1 \mathrm{D}, 2 \mathrm{D}$, or 3D) provide a detailed description of the tidal hydrodynamics at the scale of the grid element size. They offer a spatially integrating view of a system, whereas our method offers a temporally integrated view of a signal, measured at one point in space but over a much larger period of time, ranging from a few years to a century or more (e.g., Jay et al. 2011). This information is usually not obtained from high-resolution numerical models. The main advantage of an approach based on regression analysis is that no terrain description is needed (topography, substrate friction, etc.), thus minimizing sensitivity to local topographic uncertainty, which is especially a problem for historical analyses. Also, the parameter calibration is completely automated and replaced by rather simple functions whose exponents are optimized to account for local topographic and frictional effects, and whose coefficients are determined from IRLS analyses. Another advantage of NS_TIDE is that it can separate the influences of discharge and oceanic tides on the resulting tidal signal, whereas this is not as straightforward with numerical models. This approach can therefore be used with confidence in rivers where no dynamical model exists, or as a complement to these models. Hence, it provides an alternate way to look at river tides. Finally, use of NS_TIDE on model output would be an excellent way to test a numerical model of a strongly nonstationary system (cf. Foreman and Henry 1989).

\section{c. Summary}

We have developed a new HA-based method for the analysis of nonstationary tides and successfully applied it to the modeling and hindcast of river tides in the LCR. We generalized traditional HA by embedding the nonstationary fluvial and tidal forcing into the HA basis functions. The model is based on an analytical solution for incident tidal waves in frictional, convergent channels (Kukulka and Jay 2003a,b) adapted to linear regression analysis. The treatment of nontidal forcing is iteratively optimized, one tidal band at a time, to provide band-specific models that best describe the local interactions of river tides. The model output is in the form of time series of subtidal and tidal properties, expressed in terms of external forcing, for each resolved tidal frequency. They are given along with their error time series, calculated from either uncorrelated or correlated noise models. The method differs from other tidal analysis tools for nonstationary tides, in that it can distinguish frequencies within a given tidal band without any loss of resolution in the time domain. This was made possible through the redefinition of the Rayleigh criterion and the decision tree for constituent selection, which account for the smearing of the constituent spectral lines and the importance of shallow-water constituents. Applied to a tide gauge $172 \mathrm{~km}$ from the ocean with very nonstationary tides, the model gave surprisingly accurate results for river tides, with a hindcast explaining $96.4 \%$ of the original signal variance and an RMSE of $0.165 \mathrm{~m}$ for an 8-yr analysis period with highly variable river flow. Although NS_TIDE maintains the compactness of HA, it has the potential to be an effective tool for the prediction of tides in tidal rivers, provided that reasonably accurate flow predictions are available. It can be extended to other problems involving nonstationary tides, provided that suitable time series are available to quantify the relevant factors altering the tide. Our MATLAB implementation of NS_TIDE is available upon request.

Acknowledgments. Work by Pascal Matte was supported by scholarships from the Natural Sciences and Engineering Research Council of Canada and from the Ministère de l'Éducation du Québec. Support for David A. Jay and Edward D. Zaron came from the National Science Foundation grant "Secular trends in Pacific tides," and from the Miller Foundation Grant to Portland State University. Tide and flow data were provided by the National Oceanic and Atmospheric Administration, 
and the U.S. Geological Survey. The authors thank Rich Pawlowicz, Bob Beardsley, and Steve Lentz for dis-

$$
h(t)=s(t)+f(t)=\left[\begin{array}{ll}
\mathbf{A}_{s} & \mathbf{A}_{f}
\end{array}\right] \times\left[\begin{array}{ll}
\mathbf{x}_{s} & \mathbf{x}_{f}
\end{array}\right]^{\mathrm{T}}
$$
tributing the T_TIDE package, which is the basis for the adaptations proposed in this paper; Yves Secretan, who provided useful input regarding code and model verification, and four anonymous reviewers, for their constructive comments on the manuscript.

\section{APPENDIX}

\section{Solution Details}

The time series of tidal heights in Eq. (6) can be rewritten in matrix form as

with basis functions $\mathbf{A}_{s}$ and $\mathbf{A}_{f}$, such as

$$
\mathbf{A}_{s}=\left[\begin{array}{ccc}
1 & Q^{p_{s}}\left(t_{1}\right) & \frac{R^{q_{s}}\left(t_{1}\right)}{Q^{r_{s}}\left(t_{1}\right)} \\
\vdots & \vdots & \vdots \\
1 & Q^{p_{s}\left(t_{m}\right)} & \frac{R^{q_{s}}\left(t_{m}\right)}{Q^{r_{s}}\left(t_{m}\right)}
\end{array}\right]
$$

$$
\begin{aligned}
& \mathbf{A}_{f}=\left[\begin{array}{cccccc}
\cos \left(\sigma_{1} t_{1}\right) & \cdots & \cos \left(\sigma_{n} t_{1}\right) & \sin \left(\sigma_{1} t_{1}\right) & \cdots & \sin \left(\sigma_{n} t_{1}\right) \\
\vdots & \cdots & \vdots & \vdots & \cdots & \vdots \\
\cos \left(\sigma_{1} t_{m}\right) & \cdots & \cos \left(\sigma_{n} t_{m}\right) & \sin \left(\sigma_{1} t_{m}\right) & \cdots & \sin \left(\sigma_{n} t_{m}\right)
\end{array}\right. \\
& Q^{p_{f}\left(t_{1}\right) \cos \left(\sigma_{1} t_{1}\right)} \quad \cdots \quad Q^{p_{f}}\left(t_{1}\right) \cos \left(\sigma_{n} t_{1}\right) \quad Q^{p_{f}\left(t_{1}\right) \sin \left(\sigma_{1} t_{1}\right)} \quad \cdots \quad Q^{p_{f}\left(t_{1}\right) \sin \left(\sigma_{n} t_{1}\right)}
\end{aligned}
$$

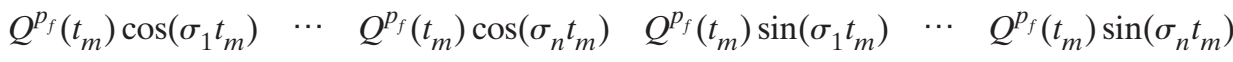

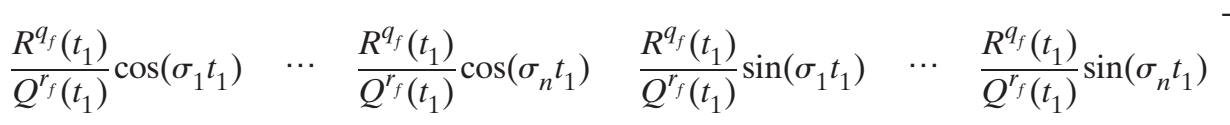

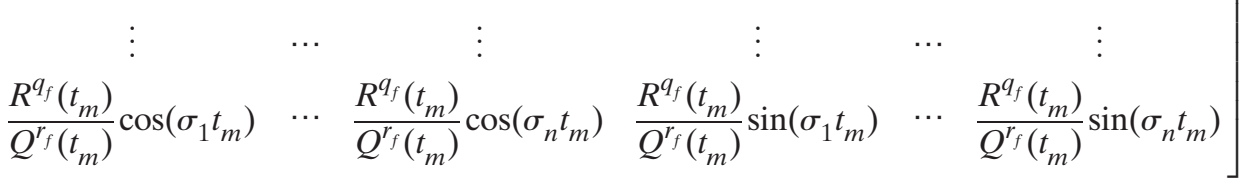

and unknown parameters $\mathbf{x}_{s}$ and $\mathbf{x}_{f}$, defined as

$$
\begin{aligned}
& \mathbf{x}_{s}=\left[\begin{array}{lll}
c_{0} & c_{1} & c_{2}
\end{array}\right] \\
& \mathbf{x}_{f}=\left[\begin{array}{llllllllllllllllll}
d_{0,1}^{(c)} & \cdots & d_{0, n}^{(c)} & d_{0,1}^{(s)} & \cdots & d_{0, n}^{(s)} & d_{1,1}^{(c)} & \cdots & d_{1, n}^{(c)} & d_{1,1}^{(s)} & \cdots & d_{1, n}^{(s)} & d_{2,1}^{(c)} & \cdots & d_{2, n}^{(c)} & d_{2,1}^{(s)} & \cdots & d_{2, n}^{(s)}
\end{array}\right] .
\end{aligned}
$$

From the coefficients $x_{s}$, the respective contribution of the freshwater discharge and the neap-spring cycle to the total stage variation can be deduced and low-frequency variation in water levels reconstructed. As for the coefficients $x_{f}$, it is useful to express each constituent $(k=1, n)$ in terms of their time-dependent amplitude and phase; hence, the expected results take the form of a time series. We define, for each term of the tidal-fluvial model, the amplitudes $A_{k}, B_{k}$, and $C_{k}$, and phases $\alpha_{k}, \beta_{k}$, and $\gamma_{k}$ for a given constituent $k$ as

$$
\begin{aligned}
& A_{k}=\sqrt{\left(d_{0, k}^{(c)}\right)^{2}+\left(d_{0, k}^{(s)}\right)^{2}}, \\
& B_{k}=Q^{p_{f}}(t) \sqrt{\left(d_{1, k}^{(c)}\right)^{2}+\left(d_{1, k}^{(s)}\right)^{2}}, \\
& C_{k}=\frac{R^{q_{f}}(t)}{Q^{r_{f}}(t)} \sqrt{\left(d_{2, k}^{(c)}\right)^{2}+\left(d_{2, k}^{(s)}\right)^{2}}, \\
& \alpha_{k}=\arctan \left(d_{0, k}^{(s)} / d_{0, k}^{(c)}\right),
\end{aligned}
$$




$$
\begin{aligned}
& \beta_{k}=\arctan \left(d_{1, k}^{(s)} / d_{1, k}^{(c)}\right), \\
& \gamma_{k}=\arctan \left(d_{2, k}^{(s)} / d_{2, k}^{(c)}\right),
\end{aligned}
$$

where $B_{k}$ and $C_{k}$ are a function of time, while the other parameters are constant. Equations (A6)-(A8) express the contribution of astronomical, river flow, and neapspring forcing to the total variance in tidal heights, respectively, while Eqs. (A9)-(A11) are their corresponding (constant) phases. To obtain the total amplitude and phase variation of a given constituent, we combine Eqs. (A6)-(A11) such that time series of amplitudes and phases are obtained. For the constituent $k$, the time series of tidal heights has the form

$$
\begin{aligned}
Z_{k}(t)= & z_{k}(t) e^{\mathrm{i} \sigma_{k} t}+z_{-k}(t) e^{-\mathrm{i} \sigma_{k} t}=\left|z_{k}(t)\right| e^{-\mathrm{i} \varphi_{k}(t)} e^{\mathrm{i} \sigma_{k} t} \\
& +\left|z_{-k}(t)\right| e^{\mathrm{i} \varphi_{k}(t)} e^{-\mathrm{i} \sigma_{k} t},
\end{aligned}
$$

where the time-dependent amplitudes and phases are given by

$$
\begin{aligned}
\left|Z_{k}(t)\right| & =\left|z_{k}(t)\right|+\left|z_{-k}(t)\right| \\
\varphi_{k}(t) & =\arctan \left\{\operatorname{Im}\left[z_{-k}(t)\right] / \operatorname{Re}\left[z_{-k}(t)\right]\right\},
\end{aligned}
$$

respectively, with, in terms of the amplitudes $A_{k}, B_{k}$, and $C_{k}$, and phases $\alpha_{k}, \beta_{k}$, and $\gamma_{k}$

$$
\begin{aligned}
z_{-k}(t)= & z_{k}^{*}(t)=\frac{1}{2}\left(A_{k} \cos \alpha_{k}+B_{k} \cos \beta_{k}+C_{k} \cos \gamma_{k}\right) \\
& +\mathrm{i} \frac{1}{2}\left(A_{k} \sin \alpha_{k}+B_{k} \sin \beta_{k}+C_{k} \sin \gamma_{k}\right) .
\end{aligned}
$$

\section{REFERENCES}

Amin, M., 1983: On perturbations of harmonic constants in the Thames estuary. Geophys. J. Roy. Astron. Soc., 73, 587-603. ,1985: Temporal variations of tides on the west coast of Great Britain. Geophys. J. Roy. Astron. Soc., 82, 279-299.

, 1993: The mutual influence of tidal constituents in the presence of bottom stress. Estuarine Coastal Shelf Sci., 37, 625-633.

Aubrey, D. G., and P. E. Speer, 1985: A study of non-linear tidal propagation in shallow inlet/estuarine systems. Part I: Observations. Estuarine Coastal Shelf Sci., 21, 185-205.

Bottom, D. L., C. A. Simenstad, J. Burke, A. M. Baptista, D. A. Jay, K. K. Jones, E. Casillas, and M. H. Schiewe, 2005: Salmon at river's end: The role of the estuary in the decline and recovery of Columbia River salmon. NOAA Tech. Memo. NMFS-NWFSC-68, 246 pp.

Buschman, F. A., A. J. F. Hoitink, M. van der Vegt, and P. Hoekstra, 2009: Subtidal water level variation controlled by river flow and tides. Water Resour. Res., 45, W10420, doi:10.1029/2009WR008167.

Byrd, R. H., J. C. Gilbert, and J. Nocedal, 2000: A trust region method based on interior point techniques for nonlinear programming. Math. Program., 89A, 149-185.
Codiga, D. L., 2011: Unified tidal analysis and prediction using the UTide Matlab functions. University of Rhode Island Graduate School of Oceanography Tech. Rep. 2011-01, $59 \mathrm{pp}$.

Daniell, P. J., 1946: Discussion of "On the theoretical specification and sampling properties of autocorrelated time-series." J. Roy. Stat. Soc., 8 (Suppl.), 88-90.

Darwin, G. H., 1891: Bakerian lecture: On tidal prediction. Philos. Trans. Roy. Soc. London, A182, 159-229.

DiLorenzo, J. L., P. Huang, M. L. Thatcher, and T. O. Najarian, 1993: Dredging impacts on Delaware estuary tides. Proceedings of the Third International Conference, M. L. Spaulding, Ed., Vol. 3, Estuarine and Coastal Modeling, ASCE, 86-118.

Doodson, A. T., 1921: The harmonic development of the tidegenerating potential. Proc. Roy. Soc. London, 100A, 305-329.

Dronkers, J. J., 1964: Tidal Computations in Rivers and Coastal Waters. North-Holland Publishing Company, 518 pp.

Foreman, M. G. G., 1977: Manual for tidal heights analysis and prediction. Pacific Marine Science Rep. 77-10, 58 pp.

— and R. F. Henry, 1989: The harmonic analysis of tidal model time series. Adv. Water Resour., 12, 109-120.

_ J. Y. Cherniawsky, and V. A. Ballantyne, 2009: Versatile harmonic tidal analysis: Improvements and applications. J. Atmos. Oceanic Technol., 26, 806-817.

Gallo, M. N., and S. B. Vinzon, 2005: Generation of overtides and compound tides in Amazon estuary. Ocean Dyn., 55, 441-448.

Godin, G., 1972: The Analysis of Tides. University of Toronto Press, 264 pp.

, 1984: The tide in rivers. Int. Hydrogr. Rev., 61, 159-170.

- 1985: Modification of river tides by the discharge. J. Waterw. Port Coastal Ocean Eng., 111, 257-274.

_ 1999: The propagation of tides up rivers with special considerations on the upper Saint Lawrence River. Estuarine Coastal Shelf Sci., 48, 307-324.

Horsburgh, K. J., and C. Wilson, 2007: Tide-surge interaction and its role in the distribution of surge residuals in the North Sea. J. Geophys. Res., 112, C08003, doi:10.1029/2006JC004033.

Huber, P. J., 1996: Robust Statistical Procedures. CBMS-NSF Regional Conference Series in Applied Mathematics, Vol. 68, 2nd ed. Society of Industrial and Applied Mathematics, 67 pp.

Jay, D. A., 1991: Green's law revisited: Tidal long-wave propagation in channels with strong topography. J. Geophys. Res., 96 (C11), 20 585-20 598.

, 2009: Evolution of tidal amplitudes in the eastern Pacific Ocean. Geophys. Res. Lett., 36, L04603, doi:10.1029/2008GL036185.

—, and E. P. Flinchem, 1995: Wavelet transform analyses of nonstationary tidal currents. Proc. Fifth Working Conf. on Current Measurement, St. Petersburg, FL, IEEE, 100-105.

$\ldots$, and _ 1997: Interaction of fluctuating river flow with a barotropic tide: A demonstration of wavelet tidal analysis methods. J. Geophys. Res., 102 (C3), 5705-5720.

— records containing multi-scale non-tidal background energy. Cont. Shelf Res., 19, 1695-1732.

- and T. Kukulka, 2003: Revising the paradigm of tidal analysis The uses of non-stationary data. Ocean Dyn., 53, 110-125.

_ , K. Leffler, and S. Degens, 2011: Long-term evolution of Columbia River tides. J. Waterw. Port Coastal Ocean Eng., 137, 182-191.

Kukulka, T., and D. A. Jay, 2003a: Impacts of Columbia River discharge on salmonid habitat: 1 . A nonstationary fluvial tide model. J. Geophys. Res., 108, 3294, doi:10.1029/2003JC001829. 
— and $\longrightarrow$, 2003b: Impacts of Columbia River discharge on salmonid habitat: 2. Changes in shallow-water habitat. J. Geophys. Res., 108, 3294, doi:10.1029/2003JC001829.

LeBlond, P. H., 1978: On tidal propagation in shallow rivers. J. Geophys. Res., 83 (C9), 4717-4721.

_ 1979: Forced fortnightly tides in shallow rivers. Atmos.Ocean, 17, 253-264.

_ 1991: Tides and their interactions with other oceanographic phenomena in shallow water (review). Tidal Hydrodynamics, B. B. Parker, Ed., Wiley, 357-378.

Leffler, K. E., and D. A. Jay, 2009: Enhancing tidal harmonic analysis: Robust (hybrid L1/L2) solutions. Cont. Shelf Res., 29, 78-88.

MathWorks, 2012: R2010a MathWorks documentation. [Available online at http://www.mathworks.com/help/releases/R2010a/ helpdesk.html.]

Munk, W. H., B. Zetler, and G. W. Groves, 1965: Tidal cusps. Geophys. J. Roy. Astron. Soc., 10, 211-219.

Nidzieko, N. J., 2010: Tidal asymmetry in estuaries with mixed semidiurnal/diurnal tides. J. Geophys. Res., 115, C08006, doi:10.1029/2009JC005864.
Parker, B. B., 1991: The relative importance of the various nonlinear mechanisms in a wide range of tidal interactions (review). Tidal Hydrodynamics, B. B. Parker, Ed., Wiley, 237-268.

- 2007: Tidal analysis and prediction. NOAA Special Publ. NOS CO-OPS 3, 378 pp.

Pawlowicz, R., B. Beardsley, and S. Lentz, 2002: Classical tidal harmonic analysis including error estimates in MATLAB using T_TIDE. Comput. Geosci., 28, 929-937.

Pugh, D. T., and J. M. Vassie, 1976: Tide and surge propagation offshore in the Dowsing region of the North Sea. Dtsch. Hydrogr. Z., 29, 163-213.

Shaw, A. G. P., and M. N. Tsimplis, 2010: The 18.6 yr nodal modulation in the tides of southern European coasts. Cont. Shelf Res., 30, 138-151.

Speer, P. E., and D. G. Aubrey, 1985: A study of non-linear tidal propagation in shallow inlet/estuarine systems. Part II: Theory. Estuarine Coastal Shelf Sci., 21, 207-224.

Waltz, R. A., J. L. Morales, J. Nocedal, and D. Orban, 2006: An interior algorithm for nonlinear optimization that combines line search and trust region steps. Math. Program., 107, 391-408. 